\title{
C60-Propylamine Adduct Monolayers at the Gas/ Water Interface: A Brewster Angle Microscopy and X-Ray Scattering Study
}

\section{Citation}

Fukumo, Masafumi, Konstantin Penanen, Ralf K. Heilmann, Peter S. Pershan, and David Vaknin. 1997. C60-propylamine adduct monolayers at the gas/water interface: A Brewster angle microscopy and x-ray scattering study. Journal of Chemical Physics 107(14): 5531-5546.

\section{Published Version}

doi:10.1063/1.474229

\section{Permanent link}

http://nrs.harvard.edu/urn-3:HUL.InstRepos:4428803

\section{Terms of Use}

This article was downloaded from Harvard University's DASH repository, and is made available under the terms and conditions applicable to Other Posted Material, as set forth at http:// nrs.harvard.edu/urn-3:HUL.InstRepos:dash.current.terms-of-use\#LAA

\section{Share Your Story}

The Harvard community has made this article openly available.

Please share how this access benefits you. Submit a story.

\section{Accessibility}




\title{
$\mathrm{C}_{60}$-propylamine adduct monolayers at the gas/water interface: A Brewster angle microscopy and $x$-ray scattering study
}

\author{
Masafumi Fukuto, Konstantin Penanen, Ralf K. Heilmann, and Peter S. Pershan \\ Department of Physics and Division of Engineering and Applied Sciences, Harvard University, Cambridge, \\ Massachusetts 02138 \\ David Vaknin \\ Ames Laboratory and Department of Physics and Astronomy, Iowa State University, Ames, Iowa 50011
}

(Received 22 May 1997; accepted 8 July 1997)

\begin{abstract}
Brewster angle microscopy (BAM), x-ray specular reflectivity and grazing-incidence $\mathrm{x}$-ray diffraction (GID) studies of $\mathrm{C}_{60}$-propylamine adduct monolayers at the gas/water interface as a function of molecular area are reported. At large molecular areas $\left(A>\sim 150 \AA^{2} /\right.$ molecule), BAM images reveal macroscopic heterogeneity in the film, consisting of the coexistence between regions covered with uniform solidlike monolayer and bare water surface. After compression to a limiting molecular area of $150 \AA^{2}$ /molecule, the film is observed to be homogeneous, with the uniform monolayer covering the entire available surface. Both the $\mathrm{x}$-ray reflectivity results and the GID patterns are consistent with the formation of a uniform monolayer at $A \sim 150 \AA^{2} /$ molecule, while the little dependence that the GID patterns have on the molecular area for $A>\sim 150 \AA^{2} /$ molecule is consistent with the heterogeneity in the film. Upon further compression to higher densities $\left(A<\sim 120 \AA^{2} /\right.$ molecule), the $\mathrm{x}$-ray reflectivity results suggest the formation of a partial layer either at the molecule/gas interface or at the molecule/water interface. In this high density regime, the shift in the observed GID pattern with molecular area is much smaller than would be expected if the film were to remain a homogeneous monolayer, also consistent with the formation of an inhomogeneous partial layer. The analysis of the broad GID pattern observed from a uniform monolayer in terms of a model 2D radial distribution function, implies a short range positional correlation, extending to only a few molecular distances. The average nearest neighbor distance $(d \sim 13 \AA)$, extracted from the GID analysis, is consistent with the limiting molecular area $\left(A \sim 150 \AA^{2} /\right.$ molecule) assuming local hexagonal packing. These results together with the sharp facets observed in the BAM images demonstrate that the monolayer when uniform is a two-dimensional amorphous solid. (C) 1997 American Institute of Physics. [S0021-9606(97)01838-2]
\end{abstract}

\section{INTRODUCTION}

One of the principal motivations behind many modern theoretical, computational, and experimental studies of surface and interfacial phenomena is to understand the effects of physical dimension on statistical physics. ${ }^{1-3}$ A principal challenge in this general area of physics has been to identify real, well defined physical systems that are suitable for experimental studies. Examples of two- or quasi-twodimensional systems that have been investigated for this purpose in recent years include rare gases adsorbed on solid substrates, ${ }^{3-6}$ freely suspended thin liquid crystal films, ${ }^{7-10}$ and Langmuir monolayers ${ }^{11,12}$ of simple long-chain molecules such as fatty acids, ${ }^{13-23}$ alcohols,${ }^{24-27}$ esters,${ }^{28}$ etc. Related statistical phenomena at surfaces and interfaces that have been often studied for more practical motivations, include wetting, ${ }^{29-31}$ premelting and reconstruction of crystalline surfaces, ${ }^{32-35}$ and surface induced order at liquid surfaces. ${ }^{36-39}$ The experimental techniques that have been applied to these various systems are as diverse as the types of systems that have been studied. It is therefore not very surprising that one of the principal applications of the contemporaniously developed synchrotron based $\mathrm{x}$-ray scattering techniques has been to probe the structure and phase transitions of both interfaces and monolayers.
The relative magnitude of the atomic cross section for elastic x-ray scattering, in comparison with the various inelastic, or absorptive processes, has given unique advantages to $\mathrm{x}$ rays for study of the bulk structure of all varieties of condensed matter. Singularly important for these purposes is the fact that with typical $\mathrm{x}$-ray wavelengths, $\lambda=1-2 \AA$, it is practical to probe structures at atomic, or molecular, length scales. The possibility of using $\mathrm{x}$ rays to study surface physics followed, in recent times, from the combination of these advantages with the enhanced intensity, high collimation and small beam size of synchrotron generated $\mathrm{x}$ rays. ${ }^{40,41}$ Nevertheless, the scattering length for $\mathrm{x}$ rays is typically many orders of magnitude larger than typical interatomic distances. Consequently the scattering length is also many orders of magnitude larger than the thicknesses of interfacial regions and the cross section for $\mathrm{x}$-ray scattering from surfaces is small. As a result of all this, most of the observed x-ray scattering from surfaces, to date, has been from ordered phases, from which coherent addition of scattering from many atoms, or molecules, gives rise to relatively sharp intense peaks that can be separated from the diffuse background scattering from other sources. ${ }^{12,34}$ To the best of our knowledge, there are only four exceptions, in which x-ray scattering can be said to have been observed from interfacial, 
or two-dimensional, systems that have only short-range order.

The first exception is that of phase transitions involving gases adsorbed within the internal atomic planes of exfoliated graphite. ${ }^{5,6}$ Several systems that have been studied exhibit a rich variety of phase transitions and critical phenomena that have been extensively modeled; however, phase transitions for these systems are complicated by the presence of the periodic graphite substrate. ${ }^{1,42}$ For example, the submonolayer behavior of $\mathrm{Kr}$ on graphite ${ }^{5}$ as well as the orderdisorder transition for $\mathrm{He}$ on graphite at a near monolayer coverage $^{4}$ can be described by "lattice gas" models, where the localized lattice gas sites reflect the long range order of the substrate. The second class of exceptions corresponds to observation of $\mathrm{x}$-ray scattering peaks from Langmuir monolayers with long-range bond-orientational order, i.e., hexatic phases, but only exponentially decaying positional order. $^{43-45}$ The observed half-widths of these peaks are broader than the resolution limited Bragg peaks observed from crystalline phases; however, the widths are still narrower than what one expects from simple liquids in which the bond-orientational order is also short range. ${ }^{46-48}$ The third example is a recent report of liquidlike order in a monolayer of $\mathrm{Bi}$ adsorbed on the surface of liquid $\mathrm{Ga} .{ }^{49}$ Finally, there are the numerous studies of the phase transitions and quasi-long-range correlations of thin films of various smectic liquid crystals. ${ }^{7,47,48,50,51}$ These are amongst the more important measurements of the structural correlations for $2 \mathrm{D}$ statistical systems; however, only the very thinnest films are strictly two dimensional.

We present here a combination of optical and x-ray scattering studies of a Langmuir monolayer (LM) of fullerenepropylamine adduct (abbreviated as C60-PA) molecules, ${ }^{52}$ which consists of a $\mathrm{C}_{60}$ molecule and twelve propylamine $\left[\mathrm{NH}_{2}\left(\mathrm{CH}_{2}\right)_{2} \mathrm{CH}_{3}\right]$ chains attached to it. The unique feature of the C60-PA system is that the number of electrons scattering coherently from any one molecule is sufficiently large that, on the basis of straightforward calculations of the x-ray structure factor of the two-dimensional Langmuir monolayer, observable scattering is predicted for phases with only short range order. We will show that the LM monolayer of C60$\mathrm{PA}$, both as-deposited and at low pressures, is a relatively incompressible solid that is either a two-dimensional amorphous glass or a two-dimensional microcrystalline solid. Although this is in itself an interesting observation, even more important is that it demonstrates that if other fullerene derivatives that form liquid, rather than solid, Langmuir monolayers could be developed, $x$-ray scattering studies of both two-dimensional solidification and vaporization would be practical. In view of the fact that the $2 \mathrm{D}$ liquid/vapor phase boundary is not well studied, this could be a major contribution to $2 \mathrm{D}$ statistical physics.

Aside from this academic interest, there is still a good deal of excitement over prospects for practical applications based on phenomena such as the electrical conductivity, and photoconductivity of fullerenes and fullerene polymers, ${ }^{53}$ for catalysis, ${ }^{54}$ and in connections with pharmaceuticals. ${ }^{55}$ In addition, there are a number of other active areas where the potential for practical application is still open; these include molecular sieves and gas storage media, and materials for nonlinear optics and superconductivity. Some of these potential applications are based around the formation of homogeneous monolayers; however, there is very little data indicating the impact that either the homogeneity of the monolayer or the degree of in-plane order, has on the phenomenology on which the applications are dependent. We believe that understanding of the physics and physical chemistry of LMs of fullerene derivatives on the surface of $\mathrm{H}_{2} \mathrm{O}$ and other liquids will serve as an important prerequisite for ordered progress in this area.

Obeng and $\mathrm{Bard}^{56}$ were amongst the first to report formation of a LM of pure $\mathrm{C}_{60}$ at the $\mathrm{H}_{2} \mathrm{O}$ /air interface. Although Maliszewskyj et al. ${ }^{57}$ support Obeng and Bard, others $^{52,58-62}$ found that the strong mutual attraction of the $\mathrm{C}_{60}$ molecules caused them to cluster, thereby destabilizing the LM. Nevertheless, Vaknin and co-workers were able to demonstrate the utility of covalent modification of $\mathrm{C}_{60}$ as a method for reducing the strong attractive interactions between clusters. ${ }^{52,62}$ The modification of $\mathrm{C}_{60}$ in this case consisted of an attachment of the multiple alkyl chains through the formation of $\mathrm{C}_{60}$-amine adducts. The peripheral alkyl chains reduce cluster-cluster interactions and enable the formation of homogeneous monolayers.

Vaknin and co-workers reported both the surface pressure/area $(\pi-A)$ isotherm of LM of the fullerenepropylamine adduct (C60-PA) mentioned above, as well as $\mathrm{x}$-ray reflectivity from the LM as a function of the area density. ${ }^{52,62}$ They observed that the reflectivity was consistent with well defined monolayers that appeared to thicken when the LM was compressed to the point where the surface pressure started to rise. The present measurements extend that work as follows: (1) We report Brewster angle microscopy (BAM) studies that allow visualization of the macroscopic morphology of the monolayer as spread and under varying degrees of compression. In fact, for a specific area greater than $\sim 150 \AA^{2} /$ molecule the surface of the water is inhomogeneously covered with regions of the LM. The LM covered regions are themselves homogeneous, having boundaries with rigid faceted edges indicating $2 \mathrm{D}$ solids. (2) Whereas the original $\mathrm{x}$-ray reflectivity measurements of Vaknin and associates used a laboratory $\mathrm{x}$-ray source, we have extended the reflectivity measurements to larger angles using synchrotron radiation. These measurements allow for a quantitative improvement in the extracted models for the surface profile. (3) We report the results of grazing incidence $\mathrm{x}$-ray diffraction measurements for varying areas/molecule that show that the solid LM-coated regions viewed by the BAM are either 2D amorphous solids (i.e., a 2D glass) or 2D microcrystalline. As judged by the angular dependence of the $\mathrm{x}$-ray scattering intensity, it appears as though the near neighbor structure of the amorphous solid changes only slightly under macroscopic compression by a factor of more than 2 .

The organization of this paper is as follows: In Sec. II, the experimental details concerning the x-ray scattering techniques, BAM, and the $\pi-A$ isotherm measurements are de- 
scribed briefly. In Sec. III, the experimental results of the $\pi-A$ isotherm, BAM, and x-ray scattering measurements on the C60-PA LM system are presented and discussed in turn. In the x-ray scattering part, the results and analysis of (1) specular reflectivity and (2) grazing-incidence diffraction and rod scans are discussed separately. Finally, a summary is given in Sec. IV, and the main conclusions from the analysis in the preceding section are highlighted.

\section{EXPERIMENTAL DETAILS}

\section{A. X-ray techniques}

The $\mathrm{x}$-ray scattering measurements reported in this paper were conducted on the Harvard/BNL liquid surface spectrometer at Beamline X22B, National Synchrotron Light Source, Brookhaven National Laboratory. A detailed description of the apparatus has been given previously. ${ }^{19}$ In the following, x-ray specular reflectivity and grazing-incidence diffraction techniques are discussed briefly.

The kinematics for the specular reflectivity technique is illustrated in Fig. 1(a). Highly collimated monochromatic $x$ rays of wavelength $\lambda$ are incident at an angle $\alpha$ to the surface. For specular reflection, the scattered $\mathrm{x}$ rays make an equal angle $(\beta=\alpha)$ to the surface within the plane of incidence, and the reflected intensity is measured as a function of the $z$ component of the wave vector transfer $Q_{z}$ $=(4 \pi / \lambda) \sin \alpha$. The component of the wave vector parallel to the surface is zero; $Q_{x y}=0 .{ }^{63}$ The critical angle $\alpha_{c}$ for total reflection is typically on the order of $0.15^{\circ}$ for water and $\lambda \sim 1.5 \AA$, and for $\alpha>4 \sim 5 \alpha_{c}$, the reflectivity $R\left(Q_{z}\right)$ is approximately given by ${ }^{12,45,64}$

$$
\frac{R\left(Q_{z}\right)}{R_{F}\left(Q_{z}\right)} \cong\left|\frac{1}{\rho_{\infty}} \int_{-\infty}^{\infty} \frac{d\langle\rho(z)\rangle}{d z} e^{i Q_{z} z} d z\right|^{2} .
$$

In the above formula, $\langle\rho(z)\rangle$ is the average electron density at some height $z$ along the surface normal, $R_{F}\left(Q_{z}\right)$ is the theoretical "Fresnel" reflectivity from an ideally flat, abrupt interface, and $\rho_{\infty}$ is the electron density in the bulk liquid, typically water. As described in the literature, measurements of $R\left(Q_{z}\right)$ can be interpreted in terms of model profiles, $\langle\rho(z)\rangle$, that describe the average electron density of the interface along the surface normal. For a homogeneous LM on the surface of water the models yield surprisingly accurate measures of the thickness of the monolayer and the average electron density. ${ }^{12,45}$ On the other hand, without an independent determination that the surface is homogeneous, such as one that can be provided by the BAM technique, it could be difficult to interpret the model profile.

Within the last several years application of grazing incidence $\mathrm{x}$-ray diffraction (GID) techniques has provided detailed information on the microscopic structure of a number of 2D phases. ${ }^{11-28,32-34}$ Except for a few cases, which have hexatic order, most of these phases exhibit resolution-limited Bragg peaks characteristic of 2D crystalline phases. Figure 1(b) contains a schematic illustration of the kinematics for the GID experiment. Highly collimated monochromatic $\mathrm{x}$ rays are incident on the surface at an angle $\alpha$ that is smaller than the critical angle $\alpha_{c}$, so that the incident beam only

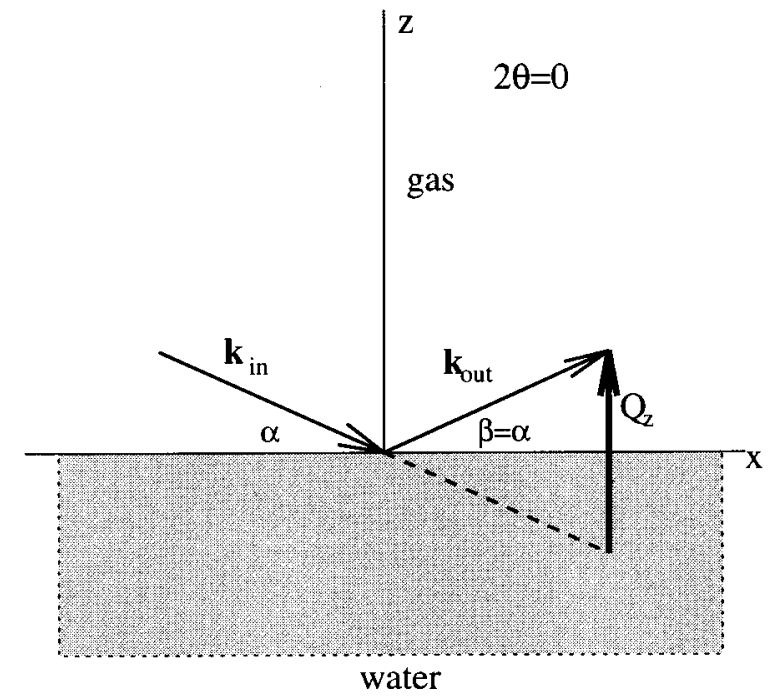

(a)

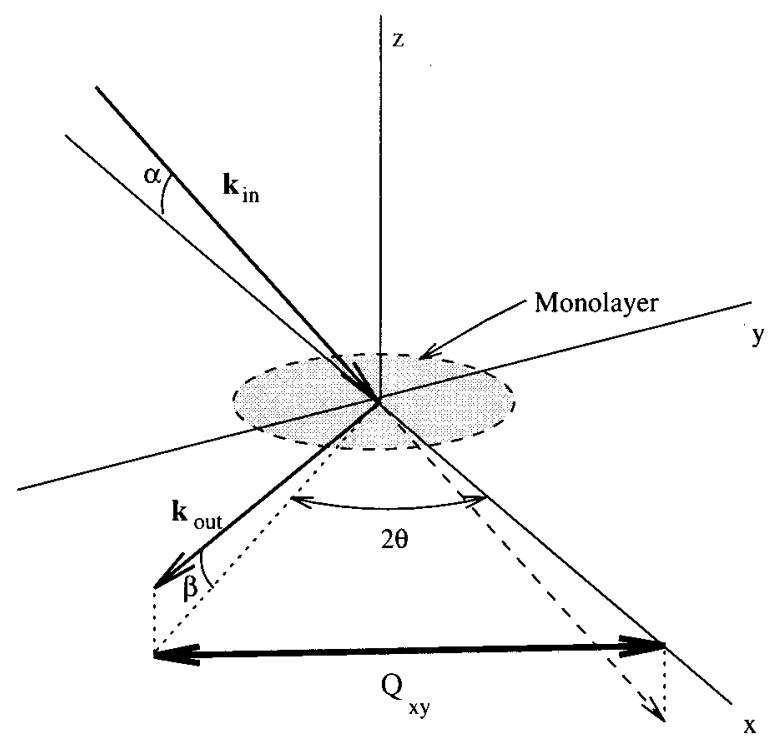

(b)

FIG. 1. Scattering geometry for (a) specular reflectivity and (b) GID.

penetrates the bulk evanescently, decaying into the bulk exponentially. Consequently for $\alpha<\alpha_{c}$ the scattering from the bulk is suppressed significantly, and given the enhanced incident flux provided by a synchrotron source, the ratio of scattering from the surface to that of the bulk is sufficient to study surface phenomena.

For GID, as illustrated in Fig. 1(b), the detector makes an angle $\beta$ to the surface. The angle $2 \theta$ is between the vertical plane containing the detected ray and the plane of incidence. In this geometry, the wave vector transfer between incident and detected radiation has components 


$$
Q_{z}=(2 \pi / \lambda)(\sin \alpha+\sin \beta),
$$

$$
Q_{x y}=(2 \pi / \lambda) \sqrt{\cos ^{2} \alpha+\cos ^{2} \beta-2 \cos \alpha \cos \beta \cos 2 \theta} .
$$

For a 2D liquid monolayer of molecules with form factor $f\left(Q_{z}, Q_{x y}\right)$ and 2D particle density of $n(r)$, the GID scattering intensity is proportional to ${ }^{65}$

$$
\begin{aligned}
S\left(Q_{x y}, Q_{z}\right) \propto & \left|f\left(Q_{z}, Q_{x y}\right)\right|^{2} \int\left\langle\left[n(0)-n_{0}\right]\left[n(r)-n_{0}\right]\right\rangle \\
& \times \exp \left(-i \mathbf{Q}_{x y} \cdot \mathbf{r}\right) d^{2} \mathbf{r} \\
\propto & \left|f\left(Q_{z}, Q_{x y}\right)\right|^{2} \int\left\{\langle n(0) n(r)\rangle-n_{0}^{2}\right\} \\
& \times J_{0}\left(Q_{x y} r\right) r d r,
\end{aligned}
$$

where $J_{0}$ is the Bessel function of zeroth order and $n_{0}$ $=\langle n\rangle$. The difference between liquid and crystalline powder has to do with the long range order in the 2D particleparticle correlation function, $\langle n(0) n(r)\rangle-n_{0}^{2}$. For a $2 \mathrm{D}$ crystalline powder $S\left(Q_{x y}, Q_{z}\right)$ consists of a series of sharp circles at radii $Q_{x y}=2 \pi / d_{h, k}$ where $d_{h, k}$ are the $d$ spacings for the $2 \mathrm{D}$ lattice. ${ }^{66}$ Radial scans that measure intensity as a function of $2 \theta$, or alternatively of $Q_{x y}$, result in sharp peaks. For a monolayer, the only structure in the scattered intensity for rod scans, in which $Q_{z}$ is varied with $Q_{x y}$ held constant at the GID peak position, results from the molecular form factor, $f\left(Q_{z}, Q_{x y}\right)$. For small molecules, or longer ones that are normal to the surface, this is typically peaked at $Q_{z}=0$ and varies slowly on the scale of $1 / L$, where $L$ is the molecular length projected on the surface normal.

For many of the 2D crystalline systems that have been studied experimentally such as, for example, behenic acid, the molecular form factor $f\left(Q_{z}, Q_{x y}\right)$ and the Debye-Waller factor combine so that only the lowest-order Bragg peaks are observed. This is particularly true for the LM films, which are the most relevant to the present paper. For 2D liquids or hexatic phases, with finite positional correlation lengths $\xi$, these peaks should be both broader and weaker. Since the peak-height and peak width are proportional to $\xi^{2}$ and $1 / \xi$, respectively, for liquidlike monolayers in which $\xi \sim 3$ or 4 molecular radii, the peak intensity becomes vanishingly small. Although broadened peaks have been observed from 2D hexatic phases of freely suspended thin liquid crystal films, ${ }^{7,48,50}$ the evidence that they have been observed for LM is ambiguous. ${ }^{67-70}$ In any event, they would certainly not be observable for liquid LM phases of behenic acid, or similar systems. ${ }^{11,18,19,28,71,72}$

\section{B. Brewster angle microscope (BAM)}

In principle GID measurements provide the most direct information of the microscopic order of 2D phases; however, microscopic information can often provide indirect evidence for a phase, or phase transition. For example using either Brewster angle microscopy or fluorescence microscopy,
Knobler et al. have observed the macroscopic structure of both crystalline domains and macroscopic strain patterns surrounding microscopic defects. ${ }^{11,72}$ Analysis of these has often yielded information on both the microscopic structure of the phases and the nature of phase transitions. Microscopic observation to establish that the monolayer is homogeneous is a necessary prerequisite to quantitative interpretation of $\mathrm{x}$-ray specular reflectivity.

The Brewster angle microscope ${ }^{73}$ used in the present study is identical to the one described by Foster et al. ${ }^{28} \mathrm{~A}$ $p$-polarized, argon-ion laser light $(\lambda=488 \mathrm{~nm})$ is incident on the monolayer at the Brewster angle for water $\theta=53.3^{\circ}$ relative to the surface normal. The size of the illuminated sample area was approximately $5 \mathrm{~mm} \times 8.6 \mathrm{~mm}$. The presence of the monolayer on the water surface destroys the Brewster condition thereby causing nonzero reflected intensity, which makes the imaging of the illuminated monolayer surface possible. The images were focused by an achromatic lens of focal length $f=175 \mathrm{~mm}$ and were captured by a CCD camera, located approximately $1450 \mathrm{~mm}$ from the focusing lens. This setup provided a magnification of 7.3 and a resolution of $20 \mu \mathrm{m}$, and the dimensions of the images captured by the CCD camera corresponded approximately to a surface area of $0.86 \mathrm{~mm} \times 1.1 \mathrm{~mm}$ on the monolayer.

\section{Langmuir trough}

The C60-PA LM sample ${ }^{52}$ was prepared on a teflon Langmuir trough with a Wilhelmy pressure sensor, all of which were sealed in an aluminum enclosure filled with $\mathrm{N}_{2}$ gas, as described by Schwartz et al. ${ }^{19}$ Millipore Milli-Q Plus water was used as the subphase. All of the measurements reported in this paper were carried out at room temperature, typically at $22-23{ }^{\circ} \mathrm{C}$. Before each spreading of a monolayer, the cleanliness of the water surface was tested by performing a quick compression isotherm on the water surface. If the surface pressure change over a compression ratio of 4 was less than $0.1 \mathrm{dyn} / \mathrm{cm}$, the surface was considered to be clean enough for use. A monolayer was prepared by spreading a chloroform solution of C60-PA $(0.28 \mathrm{mg} / \mathrm{m} \ell)$ on water at a specific area equal to or larger than $200 \AA^{2} /$ molecule. Typically, the volume of the solution spread was about 80 $\mu \ell$. From estimated uncertainties in the concentration and the added volume of the solution and from the small variation in the available water surface area with the water height, the uncertainty in the area/molecule is estimated to be less than $4 \%$. After the spreading of the film, a low flow of $\mathrm{N}_{2}$ gas was maintained for about $30 \mathrm{~min}$ to remove the evaporated chloroform and refill the enclosure with $\mathrm{N}_{2}$ gas. Then, before starting any measurements, the $\mathrm{N}_{2}$ flow was stopped, and the film was left undisturbed for about $30 \mathrm{~min}$ for equilibration.

We have used two different methods for the $\pi-A$ isotherm measurements in this study. In relaxation isotherms, ${ }^{19}$ the monolayer was allowed to relax after each step of compression by a small area change, typically $\Delta A$ $\sim 2.5 \AA^{2} /$ molecule. While the film was being relaxed at a given fixed specific area, the surface pressure was measured 
every minute. When the change in the surface pressure over five successive measurements, i.e., over $5 \mathrm{~min}$, was less than $0.05 \mathrm{dyn} / \mathrm{cm}$, a final measurement of surface pressure was made at that area/molecule, and the next compression step was taken. In continuous isotherms, the monolayer was compressed at the rate of $2\left(\AA^{2} /\right.$ molecule $) / \mathrm{min}$ and the surface pressure was measured immediately after every $1 \AA^{2}$ change in the area/molecule.

During the x-ray measurements, the temperature of the water subphase and the C60-PA film was held at $22{ }^{\circ} \mathrm{C}$. The same procedure was followed for the preparation of the monolayer, except that high-purity He gas was used in place of $\mathrm{N}_{2}$ gas in order to reduce the background scattering from the gas above the interface. The high degree of relaxation in the high-density part of the isotherm was taken into account by compressing the film in the following way. Up to the specific area at which the $\mathrm{x}$-ray measurement was conducted, the film was compressed in steps of $\Delta A \cong 2.5 \AA^{2} /$ molecule, and after each compression step, the film was relaxed for 3 min. Once the specific area of interest was reached, the film was allowed to relax more fully. The x-ray measurement was started only after the surface pressure dropped to the value given by the relaxation isotherm. At the given specific area, the measurement was repeated at least once to make sure that there was no structural change with time.

\section{RESULTS AND DISCUSSION}

\section{A. $\pi-A$ isotherm}

A typical isotherm taken at $T=22{ }^{\circ} \mathrm{C}$ for each type of isotherm is shown in Fig. 2, with the open circles and the solid line corresponding to the relaxation isotherm and the continuous isotherm, respectively. While the entire continuous scan took slightly over $1 \mathrm{~h}$, the relaxation scan took nearly 2 days due to the long relaxation time in the high density region. The shape of the continuous $\pi-A$ curve is nearly identical to the measurements reported by Vaknin et al. ${ }^{52}$ As shown in the figure, the shapes of the two isotherms are only qualitatively similar. For specific area $A$ $>150-160 \AA^{2} /$ molecule, the surface pressure remains close to zero, although the continuous isotherm shows a gradual increase in the surface pressure as $A$ approaches $150 \AA^{2} /$ molecule. Compression past $A \approx 150 \AA^{2} /$ molecule results initially in a steep increase in the surface pressure, with a very low compressibility that is constant over $\Delta A$ $\sim 20 \AA^{2}$. The value of the limiting area/molecule at the onset of the rise in $\pi$ and the slope of the $\pi-A$ curve in this region are both well reproducible for each of the isotherms. The high degree of incompressibility in the region just below $150 \AA^{2} /$ molecule suggests that the molecules become closely packed around $150 \AA^{2} /$ molecule. This value of specific area agrees well with the expected cross-sectional area of one $\mathrm{C}_{60}-\left[\mathrm{NH}_{2}\left(\mathrm{CH}_{2}\right)_{2} \mathrm{CH}_{3}\right]_{12}$ molecule and gives a strong evidence (1) that the deposited film is a monolayer and (2) that the monolayer is uniform at $150 \AA^{2} /$ molecule. One explanation that is consistent with the fact that the surface pressure remains nearly zero for specific area greater than the limiting value, is that at low densities the monolayer is macroscopi-

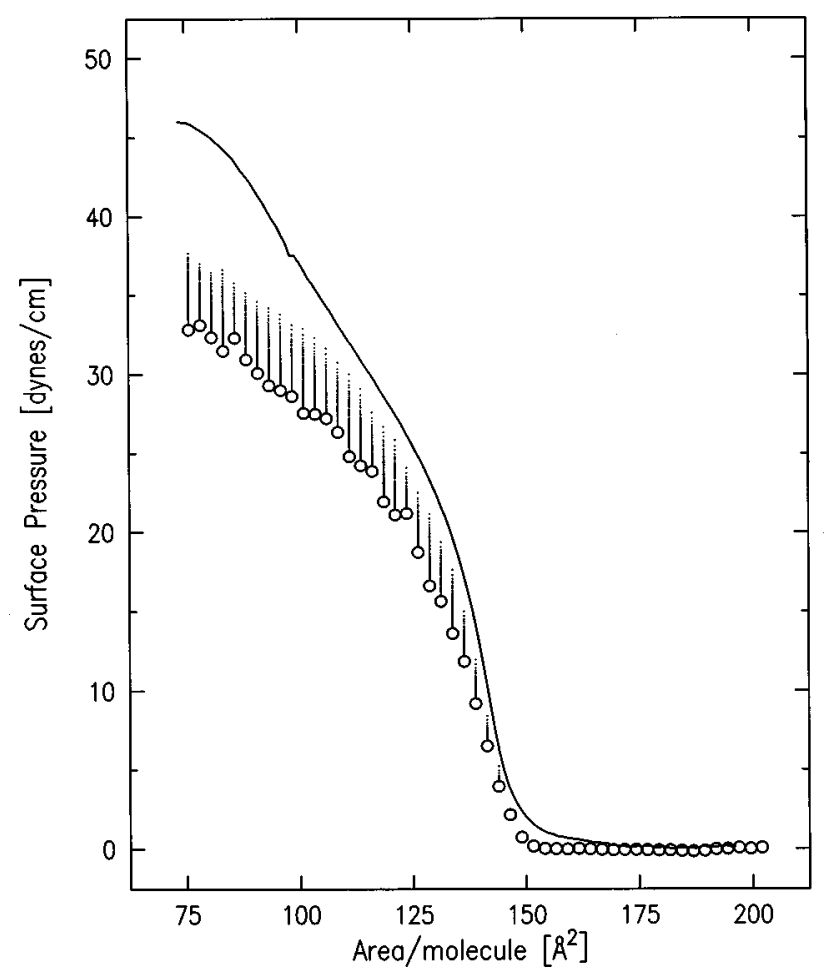

FIG. 2. $\pi-A$ isotherms taken on $\mathrm{C}_{60}$-propylamine films at $T=22{ }^{\circ} \mathrm{C}$. The solid curve (-) is a continuous isotherm, in which the film was compressed continuously at the rate of $2\left(\AA^{2} /\right.$ molecule $) /$ min. The open circles $(\bigcirc)$ correspond to the relaxation isotherm, in which the film was allowed to relax at a given fixed area/molecule until the surface pressure variation over $5 \mathrm{~min}$ was less than $0.05 \mathrm{dyn} / \mathrm{cm}$. The dots $(\cdots)$ are for the intermediate surface pressure measurements during relaxation.

cally heterogeneous and coexists with either bare water surface or a low-density 2D gas. As will be described later, the inferences made above are consistent with the BAM and reflectivity results.

In both isotherms, further compression into the highdensity region $\left(A<130 \AA^{2} /\right.$ molecule) results in a surface pressure increase with a smaller slope, where the cross-over between the two compressibilities occurs around $A$ $=125-135 \AA^{2} /$ molecule. This indicates that the film is more compressible at higher densities. A likely explanation is that in this region, compression forces the molecules out of the monolayer plane. Since the molecules have already become closely packed, a further reduction in the specific area can only be achieved either through the deformation of molecules themselves or by sending some molecules into the third dimension. The comparison between the continuous and relaxation isotherms gives a clear indication that the degree of relaxation is quite high in the high-density regimes. Although we cannot prove that the relaxed monolayer is in thermal equilibrium, it is clear that the unrelaxed monolayer is not.

\section{B. Surface imaging by BAM}

The BAM images taken on the C60-PA monolayer at various specific areas are summarized in Fig. 3. Figure 3(a) illustrates the nature of an as-deposited monolayer at specific 


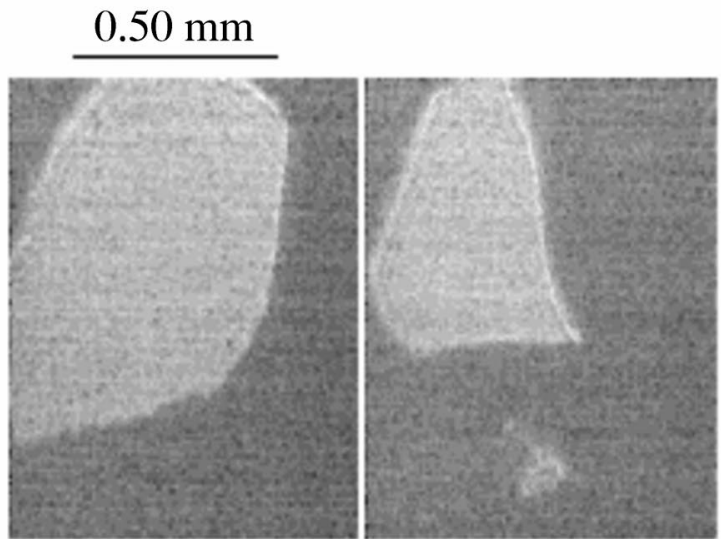

(a)

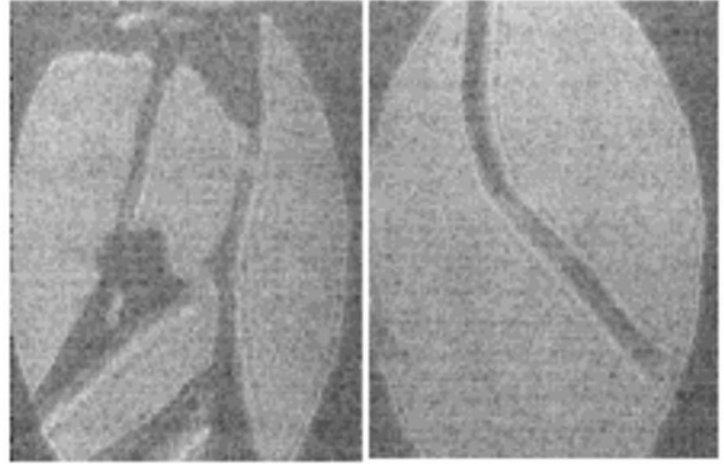

(b)
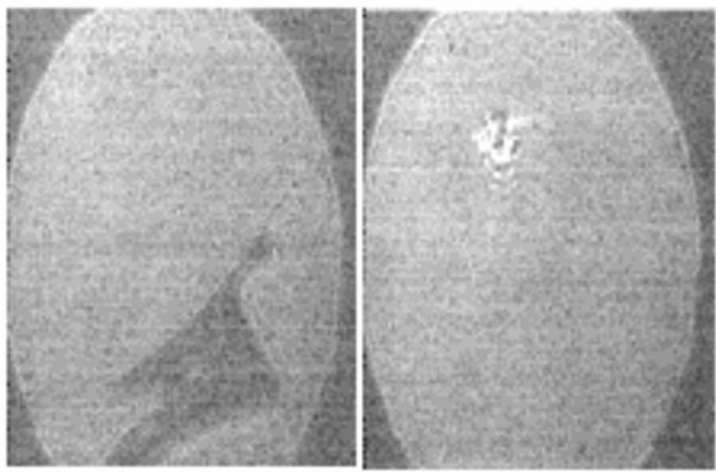

(c)
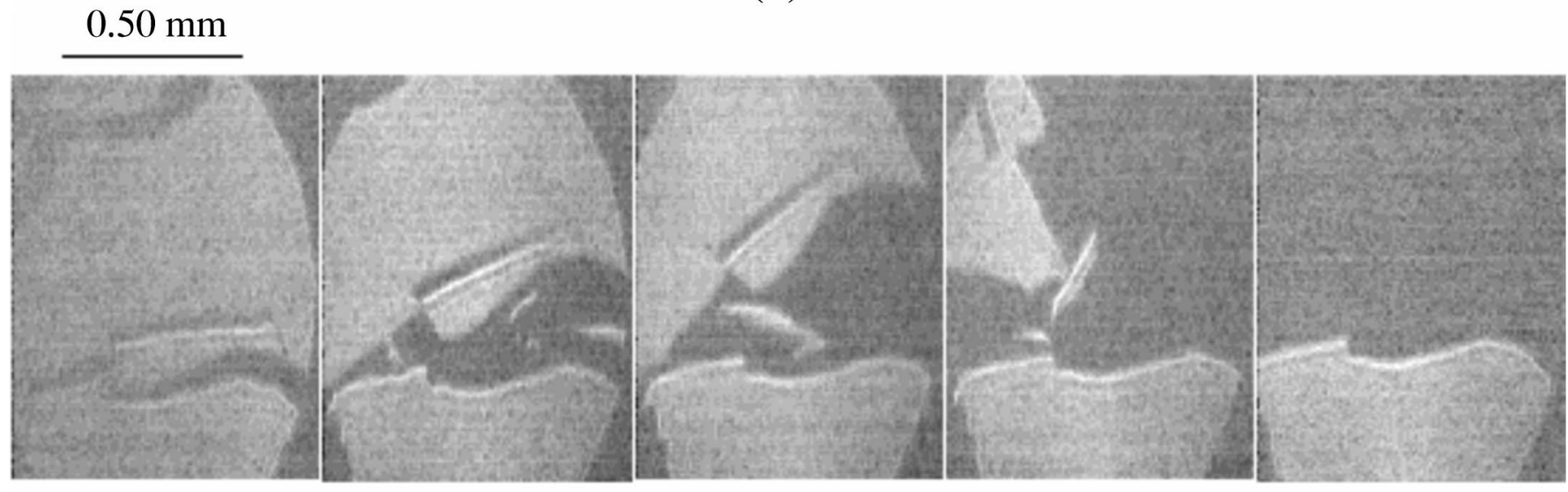

(d)

FIG. 3. BAM images taken at $A=$ (a) $280 \pm 11 \AA^{2} /$ molecule, (b) $190 \pm 8 \AA^{2} /$ molecule, and (c) $165 \pm 7 \AA^{2} /$ molecule in a compression cycle. BAM images in (d) were taken at $A=245 \pm 10 \AA^{2} /$ molecule in the expansion cycle, at $t=0,4,10,11$, and $13 \mathrm{~s}$. The film was first compressed to $165 \pm 7 \AA^{2} /$ molecule and then expanded, where the rate of compression/expansion was $\sim 0.05 \AA^{2} /$ molecule-s.

area $A=280 \pm 11 \AA^{2} /$ molecule. The coexistence of bright and dark regions is evident. At this specific area, one often finds a large uniformly bright region, which indicates a homogeneous monolayer. However, it is not difficult to find varying degrees of dark areas, which indicate either bare, uncovered water surfaces or 2D gas of C60-PA molecules at very low density. Two images in Fig. 3(a) illustrate the vary- ing degrees of surface coverage at this specific area. The relatively sharp cusp $\left(40^{\circ} \pm 5^{\circ}\right)$ formed by the boundary between the covered and bare surface is one indication that the monolayer is solid.

Upon compression, the fraction of time that the viewed area appears to be covered with a homogeneous monolayer increases. The images shown in Fig. 3(b) were taken after the 
LM was compressed continuously to a specific area of 190 $\pm 8 \AA^{2} /$ molecule at the rate of $\sim 0.05 \AA^{2} /$ molecule-s. At this specific area, homogeneous fields of view are most common. However, images like those shown in Fig. 3(b) are easy to obtain. Here too, the boundaries of the monolayer covered regions are most often straight, meeting at sharp angles that would not be expected if the monolayer were fluid.

Compression at the same rate to $165 \pm 7 \AA^{2} /$ molecule yields a monolayer for which it is very difficult to find any dark regions. Figure 3(c) illustrates two typical views of the few nonuniform regions that can be located at this specific area. Aside from being rarer, the dark regions are smaller when found. At $A \sim 150 \AA^{2} /$ molecule, it is almost impossible to locate any dark regions and, as might be implied by the isotherm, we believe that at this specific area the C60-PA LM has fully coated the surface of the trough.

According to this interpretation, further compression can only be achieved either by reducing the molecular area while maintaining an intact monolayer or by forcing some of the fullerene molecules out of the plane to form a bilayer or other multilayer structures. Although the reflected optical intensity does increase systematically with further compression, we were not able to observe any well defined contrasting regions that might have indicated macroscopically formed bilayers, or other multilayers. Such regions, if they form, must be smaller than the resolution $\sim 20 \mu \mathrm{m}$ of the BAM for weakly contrasting domains.

Figure 3(d) illustrates the process by which the uniform monolayer breaks upon expansion. This monolayer had been compressed to $165 \AA^{2} /$ molecule and then expanded at $\sim 0.05 \AA^{2} /$ molecule-s to $245 \pm 10 \AA^{2} /$ molecule, at which the images shown were observed at $t=0,4,10,11,13 \mathrm{~s}$. The shape of the boundaries when the monolayer breaks gives another strong evidence that the monolayer is solidlike.

\section{X-ray results}

\section{1. $X$-ray reflectivity}

In Fig. 4, the measured reflectivity data normalized to the theoretical Fresnel reflectivity of an ideally flat water surface, $R\left(Q_{z}\right) / R_{F}\left(Q_{z}\right)$, are shown for the $\mathrm{C}_{60}$-propylamine film at five different specific areas. From the variation of the $R\left(Q_{z}\right) / R_{F}\left(Q_{z}\right)$ curves with the specific area, it is clear that the film grows thicker with increasing density. In fitting the measured reflectivities, only the simplest models for the electron density profiles are justified because of the limited range of the $Q_{z}$ values in the data. The models we used for the average electron density profile along the surface normal are single-layer and double-layer "box" models in which each interface is smeared out with a Gaussian roughness. A single-layer model contains four free parameters and is defined as

$$
\begin{aligned}
\frac{\langle\rho(z)\rangle_{1 \text { box }}}{\rho_{\text {water }}}= & 1+(h-1) \frac{1}{2}\left[1+\operatorname{erf}\left(\frac{z+d}{\sqrt{2} \sigma_{1}}\right)\right] \\
& -h \frac{1}{2}\left[1+\operatorname{erf}\left(\frac{z}{\sqrt{2} \sigma_{0}}\right)\right],
\end{aligned}
$$

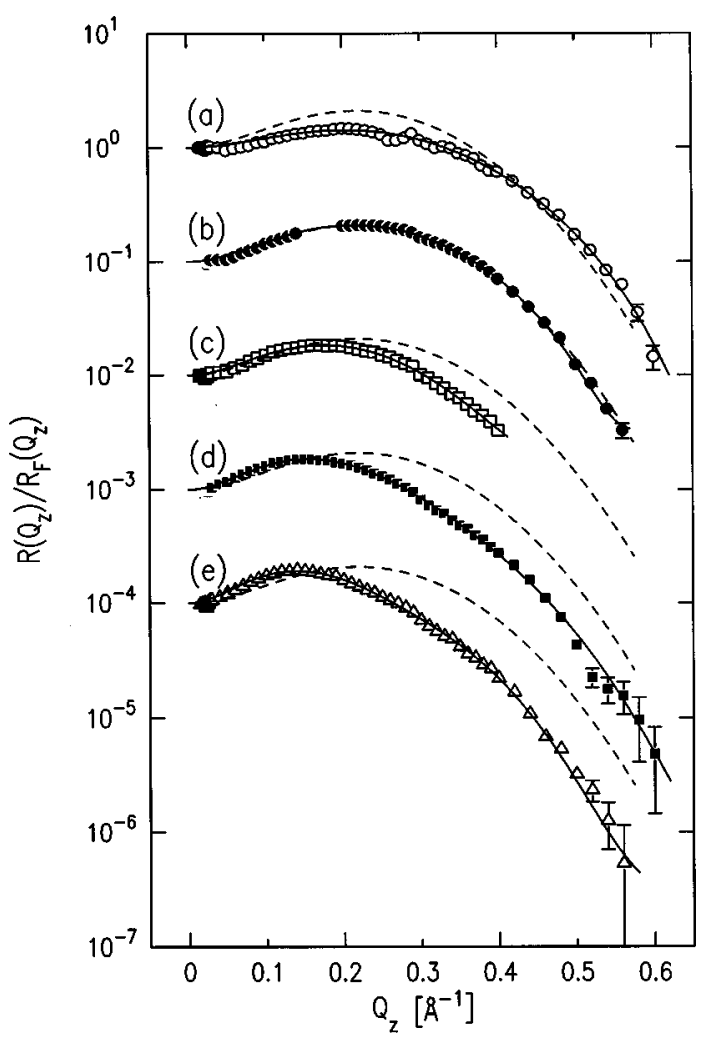

FIG. 4. Measured reflectivity normalized by the Fresnel reflectivity of an ideally flat and sharp water/gas interface, taken at $A=189 \pm 8 \AA^{2} /$ molecule (a) (O), $147 \pm 6 \AA^{2} /$ molecule (b) (๑), $126 \pm 5 \AA^{2} /$ molecule (c) ( $\left.\square\right), 105$ $\pm 4 \AA^{2} /$ molecule (d) (ロ), and $84 \pm 3 \AA^{2} /$ molecule (e) $(\triangle)$. For each, solid curve is the best fit by the box model, corresponding to the average electron density profile shown in Fig. 5. The dashed curves are all identical and correspond to the best fit to the $147 \pm 6 \AA^{2}$ data that is based on the model of average electron density profile given by the solid curve in Fig. 12(c).

where $d$ is the thickness of the monolayer, $h$ is the electron density in the layer normalized with respect to that of water $\left(\rho_{\text {water }}=0.334\right.$ electrons $/ \AA^{3}$ ), and $\sigma_{0}$ and $\sigma_{1}$ are the roughness for the gas/monolayer interface and for the monolayer/ water interface, respectively. In a double-layer model, another layer is added to the single-layer model. However, in order to keep the number of parameters small, we assumed a common thickness $d$ for both of the two layers and a common roughness $\sigma$ for all of the three interfaces. Consequently, the double-layer model also has only four free parameters and is defined as

$$
\begin{aligned}
\frac{\langle\rho(z)\rangle_{2-\text { box }}}{\rho_{\text {water }}}= & 1+\left(h_{1}-1\right) \frac{1}{2}\left[1+\operatorname{erf}\left(\frac{z+2 d}{\sqrt{2} \sigma}\right)\right] \\
& \times\left(h_{2}-h_{1}\right) \frac{1}{2}\left[1+\operatorname{erf}\left(\frac{z+d}{\sqrt{2} \sigma}\right)\right] \\
& -h_{2} \frac{1}{2}\left[1+\operatorname{erf}\left(\frac{z}{\sqrt{2} \sigma}\right)\right],
\end{aligned}
$$



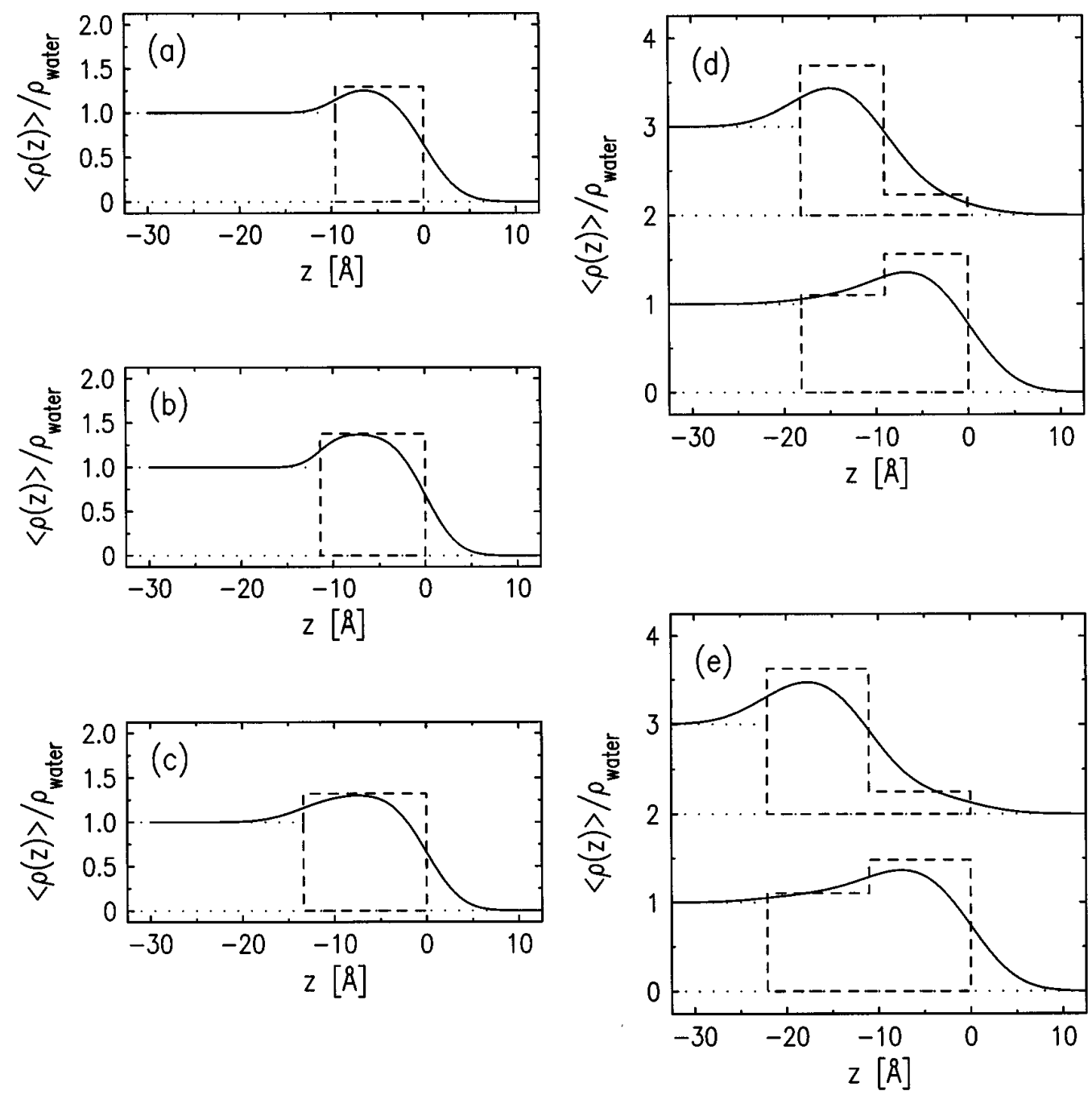

FIG. 5. The box models for the average electron density along the surface normal, normalized to the bulk density in water, all corresponding to the best fit. (a) $A=189 \pm 8 \AA^{2} /$ molecule, (b) $147 \pm 6 \AA^{2} /$ molecule, (c) $126 \pm 5 \AA^{2} /$ molecule, (d) $105 \pm 4 \AA^{2} /$ molecule, and (e) $84 \pm 3 \AA^{2} /$ molecule. The boxes in the models are indicated by the dashed lines. The dotted lines refer to the zeros and the bulk value of the electron density.

where $h_{1}$ and $h_{2}$ are the normalized electron densities in the layer just above water and in the layer just below the gas, respectively.

The nonlinear least-squares fitting to the measured $R\left(Q_{z}\right) / R_{F}\left(Q_{z}\right)$ was done using the Born approximation [Eq. (1)] and the box models just described. Since the Born approximation is valid only for $Q_{z}>4 \sim 5 Q_{c} \quad\left(Q_{c}\right.$ $=0.0218 \AA^{-1}$ for water), only the data for $Q_{z} \geqslant 0.1 \AA^{-1}$ were fitted. The best fits to $R\left(Q_{z}\right) / R_{F}\left(Q_{z}\right)$ are given by the solid curves in Fig. 4, and the corresponding average electron density profiles are shown in Figs. 5(a)-5(e) for the five specific areas. Note that for the higher density monolayers in Figs. 5(d)-5(e), the data can be equally well represented by the two different profiles. ${ }^{74}$ As indicated by the boxes [dashed lines in Figs. 5(a)-5(e)] shown along with the density profiles, the data for $A=189 \pm 8,147 \pm 6$, and 126 $\pm 5 \AA^{2} /$ molecule were fitted by the single-layer model, while the double-layer models were necessary to obtain a good fit to the data for $A=105 \pm 4$ and $84 \pm 3 \AA^{2} /$ molecule. The parameters obtained in the fitting are summarized in Table I; however, since the BAM results clearly indicate that the monolayer is inhomogeneous at $189 \AA^{2} /$ molecule, ascribing a physical meaning to the parameters for this density is questionable.

The carbon cage radius of a $\mathrm{C}_{60}$ molecule is $3.55 \AA{ }^{75,76}$ and the end-to-end length of the tetrahedrally bonded N-C$\mathrm{C}-\mathrm{C}$ unit in a propylamine chain is about $4 \AA$. If each of the twelve $\mathrm{N}-\mathrm{C}_{60}$ bonds is assumed to be about $1.5 \AA$ long and to point in the radial direction, the diameter of one C60-PA molecule is estimated to be about $16 \AA$. However, when the electron density of one such molecule is projected onto a $z$-axis, more than $90 \%$ of the electrons are concentrated within $|z|<5 \sim 6 \AA$. Therefore, if the film is a monolayer, the thickness $d$ of the layer obtained from the average electron density profile is expected to be $10-12 \AA$. As listed in Table I, the single-layer model gives $d \approx 9.6 \AA$ at $A=189$ $\AA^{2} /$ molecule and $d \approx 11.4 \AA$ at $A=147 \AA^{2} /$ molecule, and it is clear that the film is a monolayer at these specific areas. This result is consistent with the isotherm studies, and agrees with the earlier reflectivity study on the same system by 
TABLE I. The list of best-fit parameters used to fit the measured $R / R_{F}$ data, where the fits are based on Gaussian-smeared "box" models for the average electron density profile $\langle\rho(z)\rangle$ across the water/LM/gas interface. (a) Singlebox model. The thickness of and the excess electron density (relative to the bulk electron density $\rho_{\text {water }}$ ) in the monolayer are given, respectively, by $d$ and $h$. The roughness $\sigma_{0}$ is for the monolayer/gas interface, and $\sigma_{1}$ is for the water/monolayer interface. (b) and (c) Double-layer box models. Each of the two layers is assumed to have the same thickness $d$, and each of the three interfaces is assumed to have the same roughness $\sigma$. The relative electron densities $h_{1}$ and $h_{2}$ are, respectively, for the bottom layer (in contact with water) and for the top layer (in contact with vapor). The second, less dense layer is introduced as the bottom layer in part (b) and as the top layer in part (c).

(a) Single-box model

\begin{tabular}{lcrcc}
$\begin{array}{c}A^{\text {a }} \\
\left(\AA^{2} / \text { molec. }\right)\end{array}$ & $h=\rho_{\text {box }} / \rho_{\text {water }}$ & $\begin{array}{c}d \\
(\AA)\end{array}$ & $\begin{array}{c}\sigma_{0} \\
(\AA)\end{array}$ & $\begin{array}{c}\sigma_{1} \\
(\AA)\end{array}$ \\
\hline $189 \pm 8$ & $1.29 \pm 0.07$ & $9.6 \pm 0.9$ & $3.07 \pm 0.4$ & $2.09 \pm 0.7$ \\
$147 \pm 6$ & $1.38 \pm 0.05$ & $11.4 \pm 0.8$ & $2.76 \pm 0.3$ & $1.78 \pm 0.5$ \\
$126 \pm 5$ & $1.32 \pm 0.09$ & $13.4 \pm 1.8$ & $2.99 \pm 0.5$ & $3.33 \pm 0.7$
\end{tabular}

(b) Double-layer box model with the second layer below the monolayer $A^{\mathrm{a}}$

\begin{tabular}{lllcc}
\multicolumn{1}{c}{$\left(\AA^{2} /\right.$ molec. $)$} & \multicolumn{1}{c}{$h_{1}$} & $h_{2}$ & $(\AA)$ & $(\AA)$ \\
\hline $105 \pm 4$ & $1.10 \pm 0.05$ & $1.56 \pm 0.07$ & $9.1 \pm 1.2$ & $4.05 \pm 0.14$ \\
$84 \pm 3$ & $1.105 \pm 0.024$ & $1.483 \pm 0.036$ & $11.1 \pm 1.0$ & $4.03 \pm 0.12$
\end{tabular}

(c) Double-layer box model with the second layer above the monolayer $\begin{array}{lllll}105 \pm 4 & 1.69 \pm 0.09 & 0.23 \pm 0.09 & 9.1 \pm 1.2 & 4.05 \pm 0.14\end{array}$ \begin{tabular}{lllll}
$84 \pm 3$ & $1.63 \pm 0.05$ & $0.25 \pm 0.04$ & $11.1 \pm 1.0$ & $4.02 \pm 0.12$ \\
\hline
\end{tabular}

${ }^{a}$ Note that the area/molecule $A$ is not a fitting parameter.

Vaknin et al. ${ }^{52}$ Also note that the roughnesses $\sigma_{0}$ and $\sigma_{1}$ are smaller and the excess electron density $h$ is higher at 147 $\AA^{2} /$ molecule than at $189 \AA^{2} /$ molecule. Both the reduction in the roughnesses and the enhancement in the excess electron density, upon compression from 189 to $147 \AA^{2} /$ molecule, are consistent with the BAM observation that the monolayer is macroscopically inhomogeneous at $A \sim 190 \AA^{2} /$ molecule and also suggest the formation of a uniform monolayer around $150 \AA^{2} /$ molecule. At $A=126 \AA^{2} /$ molecule, the data can still be fitted by the single-layer model, but both the roughness and the thickness of the film are slightly greater than those at $147 \AA^{2} /$ molecule. The film thickness $d$ $\approx 13.7 \AA$ is still consistent with the film being a monolayer at this specific area. However, the increased thickness and roughness may also suggest that some molecules are probably starting to be forced out of the monolayer plane.

At $A=105$ and $84 \AA^{2} /$ molecule, the film is no longer a monolayer, and the fitting at these specific areas requires the introduction of a second layer. We have considered both models in which the second layer is introduced above the original monolayer and with the second layer being below the first layer. The data at each specific area can be fitted equally well by the two models, whether the second layer is introduced above or below the monolayer, and it is not possible from these data to determine which corresponds to the actual situation. Nonetheless, it is evident from both models, as indicated by the boxes in Figs. 5(d) and 5(e), that the film consists of two layers at these specific areas, and the average total thickness of the film is about twice that of a monolayer.

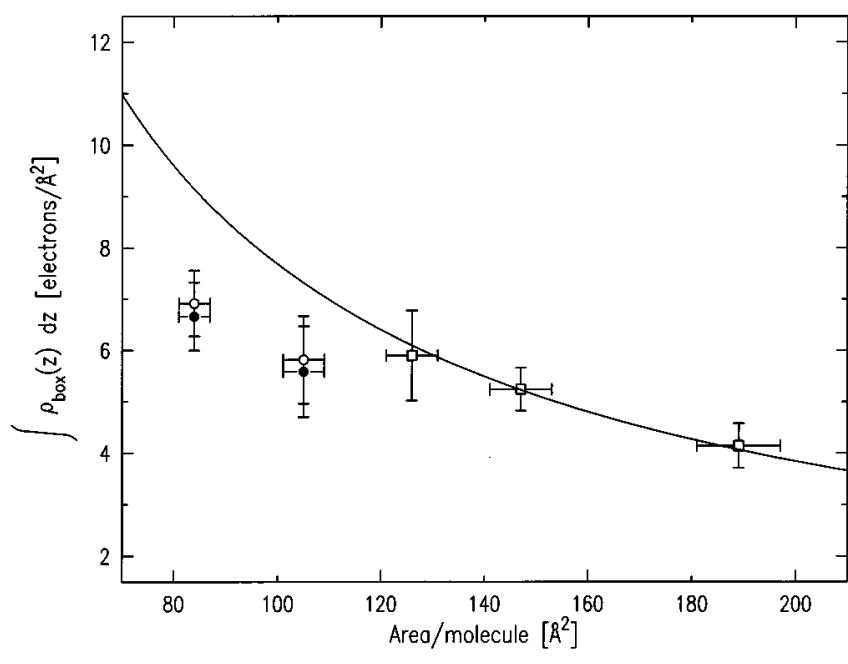

FIG. 6. The surface density of electrons from the $\mathrm{C}_{60}$-propylamine film as a function of specific area. The "measured" values are given by $\rho_{\text {water }}=0.334$ electrons $/ \AA^{3}$ times the area of the boxes in the models in Fig. 5 that corresponds to the contribution from the C60-PA molecules. The open squares $(\square)$ correspond to the single-layer models, and the circles are for the double-layer model with the second layer being above $(\bigcirc)$ and below $(\boldsymbol{O})$ the monolayer. The solid curve $(-)$ is the theoretical value, given by the number of electrons per molecule (768) divided by the specific area.

The roughness $\sigma \approx 4 \AA$ at these densities is greater than the monolayer values by $\sim 30 \%$, and this may be an indication that the second layer formed is inhomogeneous.

Since all electrons, whether from water or from C60-PA molecules, contribute to specular reflectivity in the same way in the x-ray regime, it is not strictly possible to determine the exact location of the water/film interface from the reflectivity data. However, it is reasonable, especially in the single-layer models, to use the size of the "boxes," shown in Figs. 5(a)5(e), as an estimate for the contribution from the C60-PA molecules to the average electron density. More specifically, the number of electrons from the C60-PA molecules per unit area parallel to the interface should be roughly equal to the electron density $\rho_{\text {water }}$ in water times the integrated area in the box, namely $h \cdot d$ for the single-layer model and $h_{1} \cdot d$ $+h_{2} \cdot d$ for the double-layer model with the second layer above the monolayer. In the case of the double-layer model with the second layer being below the first layer, the contribution to the box area from the C60-PA molecule should only be $x \cdot h_{2} \cdot d+h_{2} \cdot d$, where $x=\left(h_{1}-1\right) /\left(h_{2}-1\right)$ and $h_{2}$ is for the complete monolayer in this case. In Fig. 6, the surface electron density of the film calculated this way using the best-fit values is plotted as a function of specific area. Also shown in the figure (solid curve) is the "theoretical", surface electron density of the film, given by the ratio of the known number of electrons per C60-PA molecule and area/ molecule, which is a quantity completely independent of the reflectivity results. The surface electron densities in the film calculated by the two independent methods agree quite well for the monolayers at $A=189,147$, and $125 \AA^{2} /$ molecule, indicating that the values of the layer thicknesses and excess electron densities obtained from the reflectivity results are physically reasonable. However, the values of the surface 


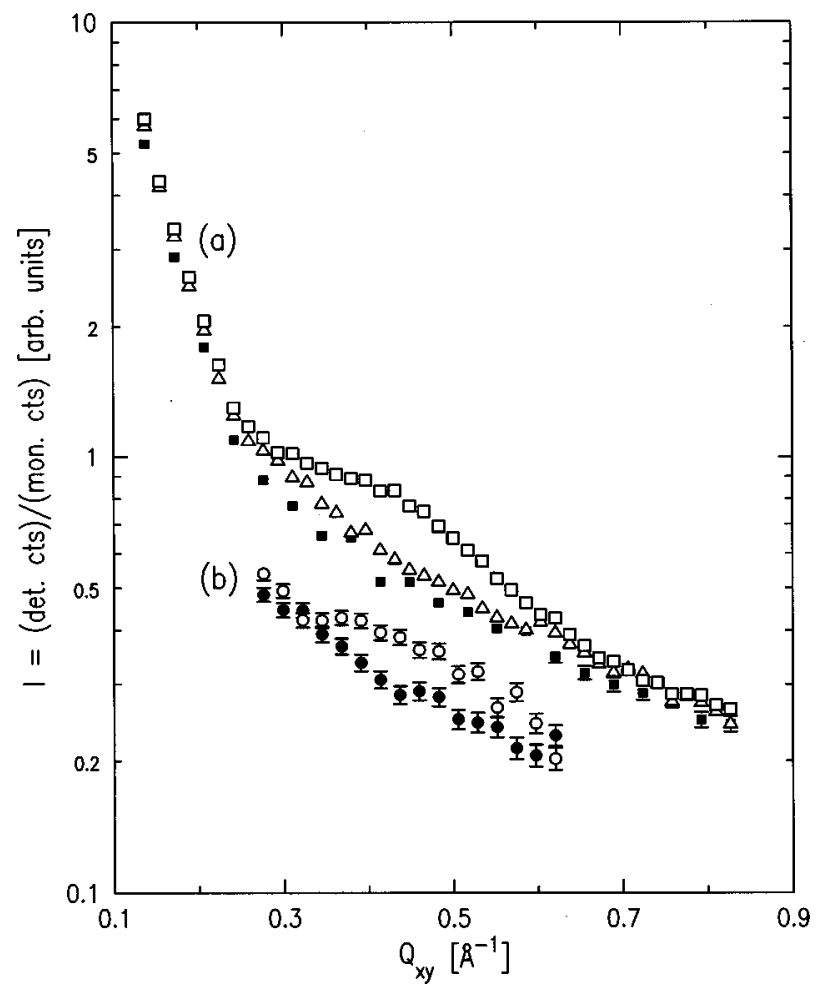

FIG. 7. (a) Typical raw GID scans taken with a set of cross slits at the detector. The three scans shown correspond to a C60-PA monolayer ( $\square$ ), water $(\triangle)$, and He background above the film (ם). (b) Typical raw GID scans taken with Soller slits. The two scans shown are for a C60-PA monolayer $(\bigcirc)$ and He background above the film $(\mathbf{O})$.

electron density based on the double-layer models are lower than the expected theoretical values. This is probably an indication that at high densities, part of the molecules that are in excess of the number needed for the complete monolayer, are collected around the barrier and edges of the trough and/or form small aggregates, although the rest of the extra molecules are forced out to a second layer as indicated by the reflectivity results.

\section{GID and rod scans}

The most important result of the GID study on the C60-PA monolayer is the observation of the x-ray scattering factor from an amorphous or liquidlike structure in two dimensions. Figure 7 illustrates typical raw GID scans of the C60-PA monolayer, clean water surface, and He gas above the monolayer. For the scans in Fig. 7(a), a single set of crossed slits of width $3 \mathrm{~mm}$ and height $20 \mathrm{~mm}$, located at distance of $605 \mathrm{~mm}$ from the sample, were used in front of the detector. Taking into account the size of the x-rayilluminated footprint on the film $(36 \mathrm{~mm} \times 0.4 \mathrm{~mm})$, the $Q_{x y}$ resolution with these slits varied from $\delta Q_{x y}=0.02$ to $0.03 \AA^{-1}$ in the range where the scans were taken. The scans shown in Fig. 7(b) were collected using Soller slits that had a horizontal acceptance angle of $3 \times 10^{-3}$ radians, corresponding to a resolution of $\delta Q_{x y}<0.012 \AA^{-1}$. In addition to those shown in the figure, scans with finer steps were taken to make certain that there was no sharp resolution-limited peak. The contribution from the C60-PA molecules to the

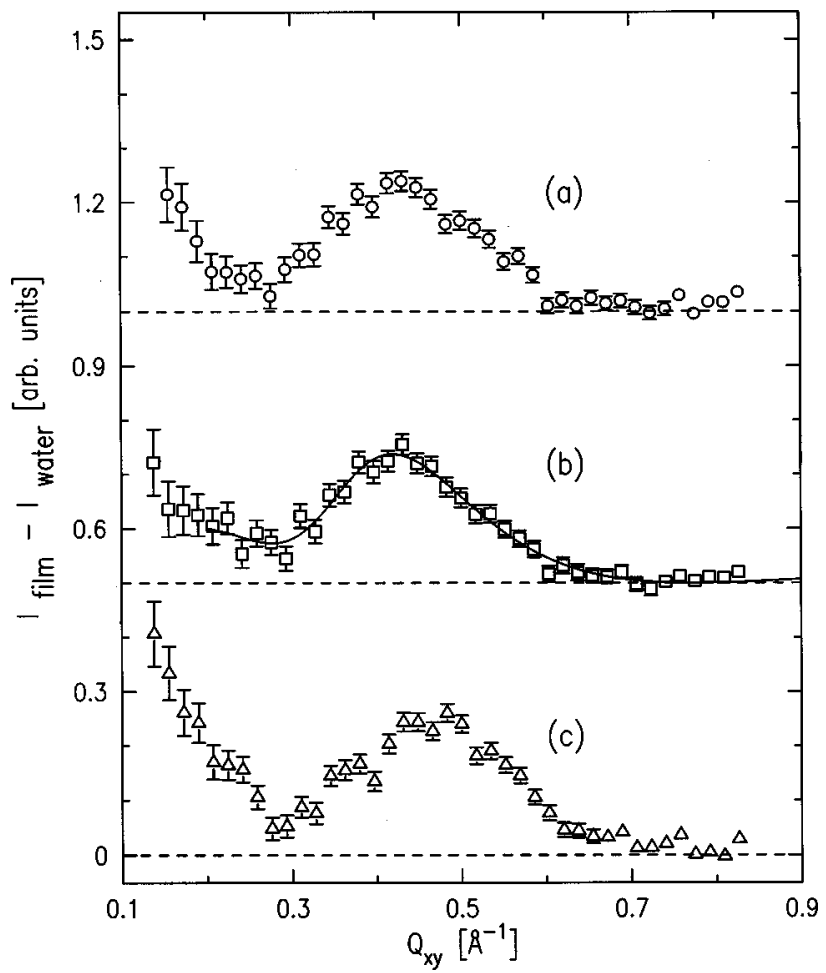

FIG. 8. The measured net GID patterns from the $\mathrm{C}_{60}$-propylamine film, after the water $+\mathrm{He}$ background has been subtracted off, for $A=$ (a) 189 $\pm 8 \AA^{2} /$ molecule, (b) $147 \pm 6 \AA^{2} /$ molecule, and (c) $105 \pm 4 \AA^{2} /$ molecule. The solid curve in (b) is the best fit at $A=147 \pm 6 \AA^{2} /$ molecule, based on the molecular form factor calculated from the spherical model of the C60-PA molecule described in Fig. 9 and the model 2D radial distribution function shown in Fig. 10.

scattered intensity is given by the difference between the raw scan on the film and that on the water surface. The result of this subtraction is shown in Fig. 8 for three specific areas, $105 \pm 4,147 \pm 6$, and $189 \pm 8 \AA^{2} /$ molecule. At all the specific areas studied, the scans from the film exhibited a broad peak which was centered at $Q_{x y}=0.42-0.45 \AA^{-1}$ and had a full width of $\Delta Q_{x y} \approx 0.2 \AA^{-1}$. The comparison between parts 7a and $7 \mathrm{~b}$ demonstrates that the peak width is much broader than and hence independent of the two detector resolutions. Consequently, the subsequent measurements were made using the coarser resolution (i.e., the regular crossed slits).

Figure 8 clearly indicates that the background subtracted GID patterns shown all have a broad peak of width $\Delta Q_{x y}$ $\approx 0.2 \AA^{-1}$. The position of the peak center at $Q_{x y}^{0}$ $\sim 0.42 \AA^{-1}$ for $A=189$ and $147 \AA^{2} /$ molecule roughly corresponds to a characteristic length of $2 \pi / Q_{x y}^{0} \sim 15 \AA$, which is comparable to the diameter of a C60-PA molecule. Since the instrumental resolution, being one order of magnitude smaller than $\Delta Q_{x y}$, is negligible, the broadness of the peak is evidence that the positional correlation of the molecules in the film is of short range. ${ }^{77}$ As discussed earlier, the reflectivity results have demonstrated that the film is a monolayer at $A=189$ and $147 \AA^{2} /$ molecule. Therefore, at these specific areas, the monolayer is a two-dimensional amorphous phase, which the BAM images have suggested is solidlike. The fact that the GID patterns at $A=189$ and $147 \AA^{2} /$ molecule are 
nearly identical, is consistent with the BAM observation that the monolayer does not fill the entire available trough area at large specific areas. Rather it consists of large "islands", which only come together to form a uniform, complete monolayer at around $150 \AA^{2} /$ molecule.

The GID pattern for $A=105 \AA^{2} /$ molecule is nearly identical to the other two except for the peak position, $Q_{x y}^{0}$ $\sim 0.45 \AA^{-1}$. This small shift is a clear indication that unlike at low densities, the compression actually pushes molecules against each other in the high-density regime, as evidenced by the increase in the surface pressure. However, the shift in the peak is much smaller than would be expected if the film were to remain homogeneous and one-molecule thick. If the compression only forced the molecules closer together in the monolayer plane, the reduction of the specific area from 147 to $105 \AA^{2} /$ molecule would correspond to the change in the average intermolecular distance by $\sim 18 \%$, and the center of the peak at $A=105 \AA^{2} /$ molecule would be at $Q_{x y}^{0}$ $\sim 0.50 \AA^{-1}$. The fact that the dependence of the GID peak position on the specific area is only slight at high densities, is consistent with the reflectivity observation that below $\sim 120 \AA 2 /$ molecule, some molecules are forced out of the monolayer plane. The explanation for the similarity of the GID patterns, aside from the slight shift in the peak position, is probably that the second layer is inhomogeneous and the observed scattered intensity comes almost entirely from the short-range positional correlation of the molecules in the first layer.

More quantitative analysis of the GID pattern has been carried out as follows. In the absence of long-range correlation, the extent of the molecular positional order in a homogeneous monolayer is characterized by a two-dimensional radial distribution function, $g(r)$, which is defined so that $n_{0} g(r) d^{2} r_{x y}$ gives the probability for finding a molecular center in $d^{2} r_{x y}$ at distance $r=\sqrt{x^{2}+y^{2}}$ given that there is another molecule at the origin. The two-dimensional average molecular density in the homogeneous monolayer is given by $n_{0}=\langle n(0)\rangle$. The $2 \mathrm{D}$ radial distribution function $g(r)$ goes to 0 as $r \rightarrow 0$ due to hard core repulsion, and approaches 1 as $r \rightarrow \infty$ since the probability is equal to the average density in the absence of correlation. The normalization condition on $g(r)$ is given by

$$
n_{0} \int_{\text {footprint }} g(r) 2 \pi r d r=N-1(\approx N),
$$

where $N$ is the number of molecules in the plane that are illuminated. In terms of the two-dimensional radial distribution function, the scattering function for the GID patterns can be expressed as ${ }^{65,78}$

$$
\begin{aligned}
S(\mathbf{Q})= & S_{0}\left|f\left(Q_{x y}, Q_{z}\right)\right|^{2} \\
& \times\left\{1+2 \pi n_{0} \int_{0}^{\infty}[g(r)-1] J_{0}\left(Q_{x y} r\right) r d r\right\},
\end{aligned}
$$

where the terms that contribute only to the scattering in the plane of incidence are omitted in the above expression. ${ }^{78,79}$ The first term on the right-hand side of Eq. (7) corresponds to the uncorrelated sum of scattering from individual mol- ecules, and the second term to the interference between the scattered waves. Apart from the number of molecules illuminated, the proportionally factor $S_{0}$ depends only on the incident angle $\alpha$, which is a fixed quantity for GID scans. Because of the normalization condition (6), the scattering function, as given by expression (7), must vanish at $Q_{x y}$ $=0$; that is, $S\left(Q_{x y}=0, Q_{z}\right)=0$.

When the entire film is a homogeneous monolayer, the two-dimensional average molecular density $n_{0}$ appearing in Eq. (7) is given by the inverse of the specific area $A$. Therefore, the use of Eq. (7) with the substitution $n_{0}=1 / A$ to fit the GID pattern is appropriate for the specific area $A$ $=147 \AA^{2} /$ molecule, at which we know that the film is a uniform monolayer. Ideally, the extraction of the 2D radial distribution function $g(r)$ from the data would involve taking the relative difference, or residual, between the observed GID pattern and the molecular form factor $\left|f\left(Q_{x y}, Q_{z}\right)\right|^{2}$ and inverse-Hankel transforming the result. However, in the present case, the limited range of the GID data and the fact that the molecular form factor for a C60-PA molecule is not well defined, make it less practical to attempt this direct method. Instead, the fitting of the GID pattern with the expression (7) was done by modeling both $g(r)$ and a spherically-symmetric average electron density $\rho_{1}(r)$ in a C60-PA molecule, which gives $f\left(Q_{x y}, Q_{z}\right)$ through Fourier transformation. Since the limited $Q_{x y}$ range of the GID data makes the fitting insensitive to any detailed structures, simple models are used here.

Our model for the radial distribution function is given by

$$
g(r)=\left\{\begin{array}{l}
h \exp \left[-\frac{(r-d)^{2}}{\sigma^{2}}\right] \quad \text { for } r \leqslant d \\
1+(h-1) \exp \left(-\frac{r-d}{\xi}\right) \cos [\kappa(r-d)] \\
\text { for } r \geqslant d
\end{array} .\right.
$$

The increase of $g(r)$ from the origin to the nearest neighbors is modeled by a Gaussian centered at an average nearest neighbor distance $d$. We have assumed that the width $\sigma$ of the Gaussian is sufficiently smaller than $d$, and neglected the fact that a small value of $g(r)$ at $r=0$ given by the model is not strictly zero. The results would not be significantly changed if the model were modified to make $g(0)$ identical to zero. In order to include the correlation with the next neighbors with the smallest number of parameters, an exponentially decaying cosine is used for $r \geqslant d$, where we have assumed that the positional correlation extends only a few intermolecular distances and the exact periodicity of the cosine function is not essential. Although there are five parameters $(d, h, \sigma, \xi, \kappa)$ in the model, only four of them are independent due to the normalization condition (6) on $g(r)$. Using the equivalent condition $S\left(Q_{x y}=0, Q_{z}\right)=0$, the parameter $h$ can be expressed in terms of the other four parameters,

$$
h=\frac{1}{I_{1}+I_{2}}\left(I_{2}+\frac{1}{2} d^{2}-\frac{1}{2 \pi n_{0}}\right),
$$

where 


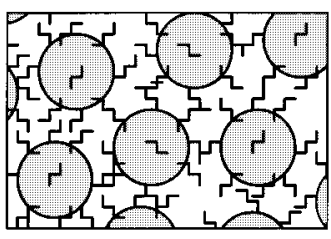

(a)

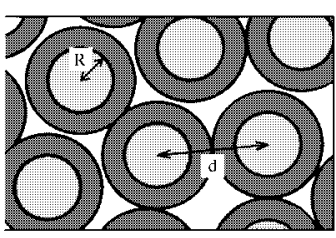

(b)

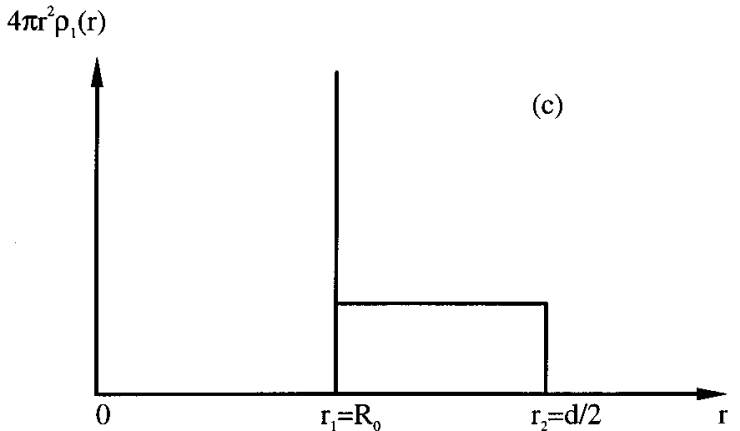

FIG. 9. (a) Illustration of a closed-packed, 2D arrangement of $\mathrm{C}_{60}$-propylamine adduct molecules on water surface. (b) Illustration for the spherically-symmetric shell model, in which the distribution of electrons in each C60-PA molecule is separated into two parts; one for the electrons from the pure $\mathrm{C}_{60}$ molecule and the other for those from the propylamine chains. (c) The model electron density within a C60-PA molecule, as a function of radius $r$. The model consists of (1) a delta function at $r_{1}=R_{0}$ $=3.55 \AA$ for the $\mathrm{C}_{60}$ molecule and (2) a spherical shell of inner radius $r_{1}$ $=R_{0}$ and outer radius $r_{2}=d / 2$ for the propylamine chains, in which electrons are distributed so that the number in each spherical shell of width $d r$ is constant

$$
\left\{\begin{array}{l}
I_{1}=\frac{\sigma^{2}}{2}\left[\frac{\sqrt{\pi} d}{\sigma} \operatorname{erf}\left(\frac{d}{\sigma}\right)+\exp \left(-\frac{d^{2}}{\sigma^{2}}\right)-1\right] \\
I_{2}=\left(\frac{\xi}{1+\kappa^{2} \xi^{2}}\right)^{2}\left[1+\frac{d}{\xi}+\kappa^{2} \xi^{2}\left(\frac{d}{\xi}-1\right)\right]
\end{array} .\right.
$$

The distribution of electrons within a C60-PA molecule consists of two parts; 360 electrons from the $\mathrm{C}_{60}$ molecule and 408 electrons from the 12 propylamine $\left[\mathrm{NH}_{2}\left(\mathrm{CH}_{2}\right)_{2} \mathrm{CH}_{3}\right]$ chains. Since the molecules in the monolayer are closely packed at $A=147 \AA^{2} /$ molecule, the propylamine chains from neighboring molecules may be intertwined, each chain may be oriented in various ways with respect to the $\mathrm{C}_{60}$ molecule it is attached to, or some chains may not be stretched out, as illustrated in Fig. 9(a). However, the observed GID data are not very sensitive to these detailed configurations, except for the number of electrons and an average size of the distribution of these electrons within one molecule. We have approximated the electron density within a molecule as spherically symmetric and consisting of two parts. The electron density corresponding to the $\mathrm{C}_{60}$ molecule is modeled as 360 electrons uniformly distributed at radius $r_{1}=R_{0}=3.55 \AA$, the known carbon-cage radius of the $\mathrm{C}_{60}$ molecule. The chains are modeled as 408 electrons distributed between $r_{1}=R_{0}=3.55 \AA$ and $r_{2}=d / 2$, one half of the average nearest-neighbor distance introduced in the model $g(r)$, in such a way that the number of electrons in
TABLE II. The list of best-fit parameters used to fit the GID pattern at $A$ $=147 \AA^{2} /$ molecule. The parameter $S_{0}$ is the proportionality factor between the observed GID intensity and the 2D structure factor. The four parameters $d, \sigma, \xi$, and $\kappa$ are defined in the model 2D radial distribution function $g(r)$ used in this paper. In the model, the increase from $g(0) \sim 0$ to $g(d)>1$ (at an average nearest neighbor distance $d$ ) is approximated as a Gaussian of width $\sigma$ centered at $r=d$, and the approach of $g(r)$ to 1 as $r$ increases is modeled as an exponentially decaying cosine, with decay length $\xi$ and angular frequency $\kappa$.

\begin{tabular}{ccccrr}
\hline \hline $\begin{array}{c}A=1 / n_{0} \\
\left(\AA^{2} / \text { molec. }\right)\end{array}$ & $\begin{array}{c}S_{0} \\
(\text { arb. })\end{array}$ & $\begin{array}{c}d \\
(\AA)\end{array}$ & $\begin{array}{c}\sigma \\
(\AA)\end{array}$ & $\begin{array}{c}\xi \\
(\AA)\end{array}$ & \multicolumn{1}{c}{$\begin{array}{c}\kappa \\
\left(\AA^{-1}\right)\end{array}$} \\
\hline $147^{\text {a }}$ & $1.474 \times 10^{-6}$ & 13.07 & 5.17 & 6.66 & 0.353 \\
& $\pm 0.045 \times 10^{-6}$ & \pm 0.38 & \pm 0.35 & \pm 0.62 & \pm 0.026 \\
\hline \hline
\end{tabular}

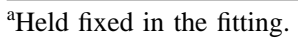

each spherical shell of width $d r$ is the same. This model is depicted in Figs. 9(b) and 9(c), and is given by

$$
\rho_{1}(r)=\left\{\begin{array}{l}
\frac{360}{4 \pi R_{0}^{2}} \delta\left(r-R_{0}\right)+\frac{408}{d / 2-R_{0}} \frac{1}{4 \pi r^{2}} \\
\text { for } r \in\left[R_{0}, d / 2\right] \\
0 \quad \text { otherwise }
\end{array},\right.
$$

where $R_{0}=3.55 \AA$. There are no additional parameters introduced in this model. By taking the Fourier transform of Eq. (10), the molecular form factor with this model is given by

$$
f\left(Q_{x y}, Q_{z}\right)=360 \frac{\sin \left(Q R_{0}\right)}{Q R_{0}}+408 \frac{\operatorname{Si}(Q d / 2)-\operatorname{Si}\left(Q R_{0}\right)}{Q\left(d / 2-R_{0}\right)},
$$

where

$$
\left\{\begin{array}{l}
Q=\sqrt{Q_{x y}^{2}+Q_{z}^{2}} \\
\operatorname{Si}(x)=\int_{0}^{x} \frac{\sin t}{t} d t
\end{array}\right.
$$

The nonlinear least-squares fitting of the GID pattern for $A=147 \AA^{2} /$ molecule has been carried out using the models (8) and (11) in expression (7), with the constraint given by the condition (9). Since the $Q_{z}$ component of the momentum transfer vector $\mathbf{Q}$ is negligible for the GID scans, we have set $Q=Q_{x y}$ in the form factor (11). There are a total of five parameters in the fitting: the four independent parameters $(d$, $\sigma, \xi, \kappa)$ from the model $g(r)$, and an additional parameter for the proportionality constant $S_{0}$ in expression (7). The best fit to the GID pattern for $A=147 \AA^{2} /$ molecule is given by the solid curve shown in Fig. 8(b), and the five parameters corresponding to the best fit are summarized in Table II. The model $g(r)$ using these parameters is shown in Fig. 10.

As evident in Fig. 10, the positional correlation of the molecules is of short range and extends only up to the nextnearest neighbors, which demonstrates that the monolayer is indeed amorphous. The best fit gives the value of the average nearest-neighbor distance at $d=13.1 \pm 0.4 \AA$, which is comparable to the thickness of the monolayer obtained from the reflectivity measurement. It is slightly larger than the intermolecular distance of $10 \AA$ in the fcc crystal of pure $\mathrm{C}_{60},{ }^{75,76}$ but this is expected due to the presence of propylamine 


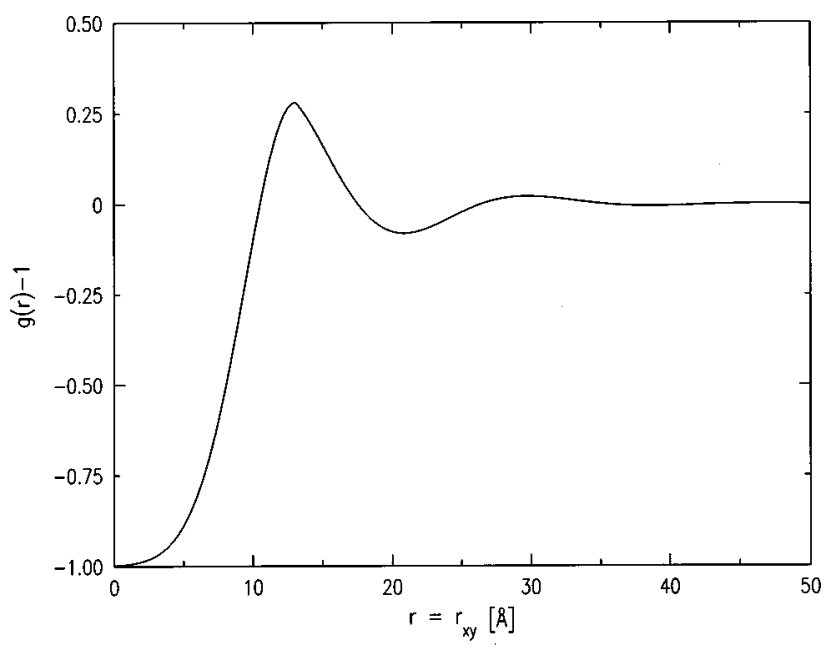

FIG. 10. The model for $g(r)-1$ as a function of $r=r_{x y}=\sqrt{x^{2}+y^{2}}$, corresponding to the best fit to the GID pattern at $A=147 \pm 6 \AA^{2} /$ molecule [see Fig. 8(b)]. $g(r)$ is the two-dimensional radial distribution function. The average nearest-neighbor distance is at $d=13.1 \pm 0.4 \AA$. In the model, the $r<d$ part of $g(r)$ is approximated by a Gaussian centered at $d$ and the $r$ $>d$ part is approximated by an exponentially decaying cosine that approaches 1 as $r$ increases to infinity.

chains. The obtained value for an average intermolecular distance, $d$, provides an independent measure for the average molecular density, or the specific area, within the uniform monolayer. Assuming that the C60-PA molecules are locally arranged by hexagonal packing, the area/molecule is given by $A=(\sqrt{3} / 2) d^{2}$. Using the value $d=13.1 \pm 0.4 \AA$ obtained from the best fit, the specific area based on the GID measurement is given by $A_{\mathrm{GID}}=149 \pm 6 \AA^{2} /$ molecule which is in excellent agreement with the value $A_{\text {trough }}=147 \AA^{2} /$ molecule, based on the trough measurement. This result is consistent with the BAM and isotherm observations that the monolayer consists of islands at low densities and it becomes uniform at $\sim 150 \AA^{2} /$ molecule, where the molecules become closely packed over the whole available surface and the surface pressure begins to increase.

In order to obtain an independent measure for the molecular form factor, rod scans were carried out. In the rod scans, scattered intensity was scanned along the angle $\beta$ in Fig. 1(b), while keeping the angle $2 \theta$ fixed near the maximum of the GID peak. In terms of the momentum transfer vector, the procedure corresponded to scanning along $Q_{z}$ while $Q_{x y}$ was held fixed at $0.45 \AA^{-1}$. The same scans were performed on C60-PA film and He gas above the film. The difference between the scans on the water and He background was negligibly small compared to that between the film and the He background. Figure 11 shows the intensity along the rod for three specific areas $A=105 \pm 4,147 \pm 6$, and $189 \pm 8 \AA^{2} /$ molecule, after subtracting the corresponding contribution from the He background. As expected for spherically symmetric molecules, the rods are centered about $Q_{z}=0$.

The scattering function for the rod scan corresponds to the $Q_{z}$ dependence of the scattering function of the GID scan with $Q_{x y}$ held fixed at a peak position. Taking into account

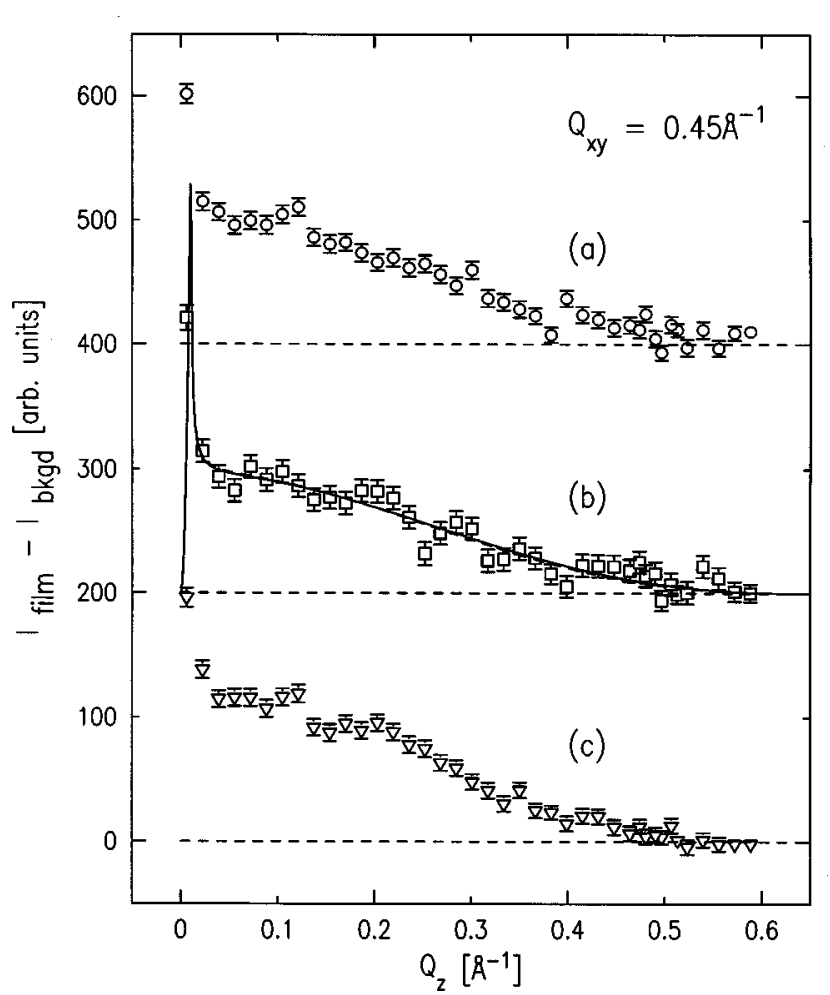

FIG. 11. Measured rod scans at $Q_{x y}=0.45 \AA^{-1}$ (fixed) as a function of $Q_{z}$, taken at $A=$ (a) $189 \pm 8 \AA^{2} /$ molecule, (b) $147 \pm 6 \AA^{2} /$ molecule, and (c) $105 \pm 4 \AA^{2} /$ molecule. The solid curve in (b) is the best fit at $A=147 \pm 6$ $\AA^{2} /$ molecule, assuming that the molecular form factor is given by the spherical model of the C60-PA molecule described in Fig. 9. The dashed line, which is nearly indistinguishable from the solid line, is the result of the fitting when the outer radius $r_{2}$ of the C60-PA molecule is fixed at the value $r_{2}=6.54 \AA$ extracted from the GID analysis.

the surface enhancement factor $T(\alpha) T(\beta)$, where $T(\alpha)$ $=\left(2 \alpha / \alpha_{c}\right)^{2} \sqrt{R_{F}(\alpha)}$, and noting that the incident angle is fixed, ${ }^{34,71}$

$$
S_{\mathrm{Rod}}\left(Q_{z}\right)=S_{1} T(\beta)\left|f\left(Q_{x y}^{0}, Q_{z}\right)\right|^{2},
$$

where $Q_{x y}^{0}=0.45 \AA^{-1}$ and $S_{1}$ is a proportionality constant. The data for $A=147 \pm 6 \AA^{2} /$ molecule has been fitted using Eqs. (11) and (12), with $S_{1}$ and $d$ as the only parameters. The best fit to the observed rod scan is given by the solid curve in Fig. 11(b). The fit gives the value of the outer radius in the $\rho_{1}(r)$ model at $r_{2}=d / 2=6.17 \pm 0.6 \AA$, or $d=12.4 \pm 1.2 \AA$, which agrees fairly well with the value obtained in the GID analysis. The dashed line, which is almost completely covered by the solid line, corresponds to the fit with the outer radius fixed at the GID based value $r_{2}=d / 2=6.54 \AA$.

\section{Application of the spherical model to the fitting of reflectivity data}

Finally, we show here that the above spherical model of the C60-PA molecules can also be applied to the fitting of the reflectivity data, by constructing a model electron density profile based on the spherical model and using it to fit the reflectivity result at $A=147 \AA^{2} /$ molecule. The electron densities shown in Figs. 12(a)-12(c) summarize the basic ideas behind this particular model. First, the electron density in 

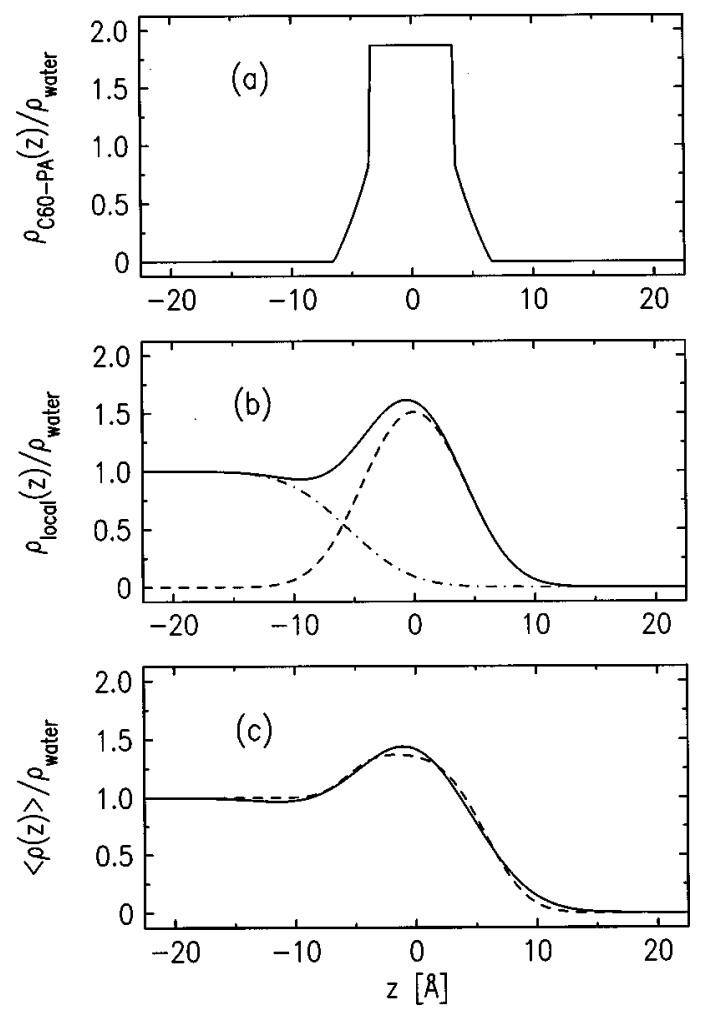

FIG. 12. A model of electron density profile based on the spherical C60-PA model (see Fig. 9). All the profiles correspond to the best fit to the reflectivity data at $147 \pm 6 \AA^{2} /$ molecule. (a) The projection onto the $z$-axis of the model electron density within one C60-PA molecule, normalized to the bulk density in water, assuming the GID-based value $r_{2}=6.54 \AA$ for the outer radius of the C60-PA molecule. (b) Models for the local electron density profile of the C60-PA monolayer (---), and of water (-.-), and of the total local electron density (-). The model for the monolayer is the convolution of the result (a) with a Gaussian of width $\sigma_{\mathrm{LM}}$. (c) The solid line is the average electron density profile, given by the convolution of the total local electron density in part (b) with a Gaussian of roughness $\sigma$ due to thermally excited capillary waves. The dashed curve is the result of the single-box model at $147 \pm 6 \AA^{2} /$ molecule, shown here for comparison.

one C60-PA molecule, assuming the spherical model with the outer radius $r_{2}=d / 2=6.54 \AA$, was projected onto the $z$-axis by integrating over $(x, y)$. Since the monolayer covers the trough surface uniformly and the molecules are closely packed at this specific area, we can attribute a column with hexagonal cross sectional area $A=(\sqrt{3} / 2) d^{2}$ to each molecule. The contribution from the C60-PA molecule to the electron density in this column is given by dividing the projected one-molecule density by $(\sqrt{3} / 2) d^{2}$, which is denoted as $\rho_{\mathrm{C} 60-\mathrm{PA}}(z)$ in Fig. 12(a). Now assuming that the local distribution for the heights of molecular centers is Gaussian with a characteristic width $\sigma_{\mathrm{LM}}$, the contribution from the monolayer to the local electron density is given by the convolution of $\rho_{\mathrm{C} 60-\mathrm{PA}}(z)$ with this Gaussian distribution. This is shown by the dashed curve in Fig. 12(b). The contribution from the water to the local density was modeled with a simple error function, located at distance $-l$ into the bulk and having a width $\sigma_{\text {water }}$. The sum of the two contributions gives the total local electron density, which is shown by the solid curve in Fig. 12(b). Finally, the local electron density was convoluted with the roughness $\sigma$ due to the thermally
TABLE III. The list of best-fit parameters used to fit the $R / R_{F}$ data measured at $A=147 \AA^{2} /$ molecule, where the fitting is based on a model average electron density profile $\langle\rho(z)\rangle$ calculated assuming a spherically symmetric electron density within the $\mathrm{C}_{60}$-propylamine adduct molecule. The outer radius $r_{2}$ of the C60-PA molecule was held fixed at one half of the average nearest neighbor distance $d$ extracted from the GID analysis. The model assumes that the local height distribution of the C60-PA molecules is Gaussian of width $\sigma_{\mathrm{LM}}$, and that the water/monolayer interface has a width $\sigma_{\text {water }}$ and is located at distance $-l$ below the average height of molecular centers. Since the surface pressure is close to zero at $A=147 \AA^{2} /$ molecule, the roughness $\sigma$ due to thermally excited capillary waves was held fixed at the value measured for clean water surface.

\begin{tabular}{lcccc}
\hline \hline $\begin{array}{l}r_{2} \\
(\AA)\end{array}$ & $\begin{array}{c}\sigma_{\mathrm{LM}} \\
(\AA)\end{array}$ & $\begin{array}{c}\sigma_{\text {water }} \\
(\AA)\end{array}$ & $\begin{array}{c}-l \\
(\AA)\end{array}$ & $\begin{array}{c}\sigma \\
(\AA)\end{array}$ \\
\hline $6.54^{\mathrm{a}}$ & $3.08 \pm 0.12$ & $4.09 \pm 0.45$ & $-5.42 \pm 0.23$ & $2.60^{\mathrm{a}}$ \\
\hline \hline
\end{tabular}

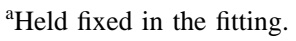

excited capillary waves,${ }^{80}$ to obtain the average electron density profile, shown by the solid curve in Fig. 12(c). Since the surface pressure at this specific area is close to zero, we have set the roughness at the value $\sigma=2.60 \AA$ of the clean bare water surface, which was measured with the same experimental resolution prior to the spreading of the monolayer. With this model, the only free parameters in the fitting are $\sigma_{\mathrm{LM}}, \sigma_{\text {water }}$, and $l$. The best fit to the reflectivity data at $A$ $=147 \AA^{2} /$ molecule is given by the dashed curves in Fig. 4 . The density profiles shown as solid lines in Fig. 12 correspond to the best-fit values of the three parameters, which are listed in Table III

The average electron density profile obtained with the above spherical model for the C60-PA molecule is in a qualitative agreement with the one obtained earlier by the singlebox model. The thickness and the height of the excess electron density in the monolayer are similar in the two models, as compared in Fig. 12(c). This again shows that the film at this specific area is a homogeneous monolayer with density just right to cover the entire available surface with closely packed molecules. On the other hand, we also note a small difference in the shapes of the two profiles. The detailed features in the profiles are highly dependent on the models used, and it is difficult to distinguish the two models simply from the reflectivity results because of the limited range and accuracy in the data. This is an example of the limitation on the extent over which any detailed features can be extracted from a given reflectivity result.

\section{SUMMARY}

Using Brewster angle microscopy and $\mathrm{x}$-ray scattering techniques, both the macroscopic and microscopic structure of $\mathrm{C}_{60}$-propylamine adduct monolayers on water have been studied at various surface densities. At low densities $(A>$ $\sim 150 \AA^{2} /$ molecule), the monolayer is macroscopically heterogeneous, with the surface consisting of regions covered with a uniform solidlike monolayer and regions of bare water surface. The compression at these densities only reduces the area of uncovered surface, until the monolayer becomes macroscopically uniform at $A \sim 150 \AA^{2} /$ molecule. 
This behavior is evidenced by (1) the BAM images and the following results: (2) the surface pressure remains zero until it begins to increase at $A \sim 150 \AA^{2} /$ molecule, (3) both the reflectivity and GID results are consistent with the formation of a closely packed, uniform monolayer at $A \sim 150 \AA^{2} / \mathrm{mol}$ ecule, and finally (4) the GID patterns at low densities are essentially identical to the one at $A \sim 150 \AA^{2} /$ molecule. For high densities $\left(A<\sim 120 \AA^{2} /\right.$ molecule $)$, the compression forces out of the monolayer plane those molecules that are in excess of the number needed for a complete monolayer, with some of the molecules forming 3D aggregates and/or collected around the barrier and edges of the trough and the rest going to a second layer above or below the original monolayer. The main evidences for this behavior are (1) the model electron density profiles obtained from the reflectivity results at high densities and (2) the fact that the shift in the position of the GID peak at high densities is much smaller than would be expected if the film were to remain a homogeneous monolayer at high densities.

The most important result of this study on the $\mathrm{C}_{60}$-propylamine adduct monolayer on water is the experimental observation of the x-ray scattering factor from a twodimensional structure with only a short-range positional order. By constructing a model $2 \mathrm{D}$ radial distribution function $g(r)$ and utilizing it to fit the observed GID pattern at $A$ $=147 \AA^{2} /$ molecule, we have shown that the positional correlation of the molecules in the uniform monolayer extends only over a few molecular distances. While this result is interesting in its own right, it is also important to note that the observation of such a $2 \mathrm{D}$ amorphous structure was made possible mainly by the large number of electrons contained in each scattering unit, the $\mathrm{C}_{60}$-propylamine adduct molecule in the present case. To the best knowledge of the authors, the $\mathrm{x}$-ray GID studies of Langmuir monolayers have so far been limited to hexatic phases and crystalline structures with quasilong range positional order. Considering this, the results of the present study have an important implication that if other $\mathrm{C}_{60}$ derivatives can be developed that (1) form stable monolayers on water and (2) have an order-disorder transition with a liquid as the disordered phase, then, it may be possible to study both sides of these transitions with the $\mathrm{x}$-ray scattering techniques.

\section{ACKNOWLEDGMENTS}

The Harvard contribution to this research has been supported by Grant Nos. NSF-DMR-95-23440 and NSF-DMR94-00396. Ames Laboratory is operated for the U.S. Department of Energy by Iowa State University under Contract No. $\mathrm{W}-7405$-Eng-82. Some of the experiments were carried out on the Harvard/BNL liquid surface reflectometer on beamline X22B at the National Synchrotron Light Source, Brookhaven National Laboratory. This facility is supported by DE-AC02-76CH00016.

\footnotetext{
${ }^{1}$ D. R. Nelson, in Phase Transitions and Critical Phenomena, edited by C. Domb and J. L. Lebowitz (Academic, New York, 1983), Vol. 7, pp. 1-99.

${ }^{2}$ K. J. Strandburg, Rev. Mod. Phys. 60, 161 (1988).
}

${ }^{3}$ Ordering in Two Dimensions, edited by S. K. Sinha (North-Holland, Amsterdam, 1980).

${ }^{4}$ R. Marx, Phys. Rep. 125, 1 (1985).

${ }^{5}$ R. J. Birgeneau and P. M. Horn, Science 232, 329 (1986).

${ }^{6}$ K. Knorr, Phys. Rep. 214, 113 (1992).

${ }^{7}$ P. S. Pershan, Structure of Liquid Crystal Phases (World Scientific, Singapore, 1988).

${ }^{8}$ J. B. Brock, R. J. Birgeneau, J. D. Litster, and A. Aharony, Physics Today July, 52 (1989).

${ }^{9}$ B. D. Swanson, H. Stragier, D. J. Tweet, and L. B. Sorensen, Phys. Rev. Lett. 62, 909 (1989).

${ }^{10}$ C. Bahr, Int. J. Mod. Phys. B 8, 3051 (1994).

${ }^{11}$ C. M. Knobler, in Advances in Chemical Physics, edited by S. Rice and I. Prigogine (Wiley, New York, 1990), Vol. 77, p. 397.

${ }^{12}$ J. Als-Nielsen, D. Jacquemain, K. Kjaer, F. Leveiller, M. Lahav, and L. Leiserowitz, Phys. Rep. 246, 251 (1994).

${ }^{13}$ M. K. Durbin, A. Malik, R. Ghaskadvi, M. C. Shih, P. Zschack, and P. Dutta, J. Phys. Chem. 98, 1753 (1994).

${ }^{14}$ I. R. Peterson, R. M. Kenn, A. Goudot, P. Fontaine, F. Rondelez, W. G. Bouwman, and K. Kjaer, Phys. Rev. E 53, 667 (1996).

${ }^{15}$ B. Lin, M. C. Shih, T. M. Bohanon, G. E. Ice, and P. Dutta, Phys. Rev. Lett. 65, 191 (1990).

${ }^{16}$ M. C. Shih, T. M. Bohanon, J. M. Mikrut, P. Zschack, and P. Dutta, Phys. Rev. A 45, 5734 (1992).

${ }^{17}$ R. M. Kenn, C. Böhm, A. M. Bibo, I. R. Peterson, H. Möhwald, K. Kjaer, and J. Als-Nielsen, J. Phys. Chem. 95, 2092 (1991).

${ }^{18}$ G. M. Bommarito, W. J. Foster, P. S. Pershan, and M. L. Schlossman, J. Chem. Phys. 105, 5265 (1996).

${ }^{19}$ D. K. Schwartz, M. L. Schlossman, and P. S. Pershan, J. Chem. Phys. 96, 2356 (1992).

${ }^{20}$ V. M. Kaganer, I. R. Peterson, R. M. Kenn, M. C. Shih, M. Durbin, and P. Dutta, J. Chem. Phys. 102, 9412 (1995).

${ }^{21}$ G. A. Overbeck and D. Möbius, J. Phys. Chem. 97, 7999 (1993).

${ }^{22}$ S. Rivière, S. Hénon, J. Meunier, D. K. Schartz, M.-W. Tsao, and C. M. Knobler, J. Chem. Phys. 101, 10045 (1994).

${ }^{23}$ S. Rivière-Cantin, S. Hénon, and J. Meunier, Phys. Rev. E 54, 1683 (1996).

${ }^{24}$ S. W. Barton, B. N. Thomas, E. B. Flom, S. A. Rice, B. Lin, J. B. Peng, J. B. Ketterson, and P. Dutta, J. Chem. Phys. 89, 2257 (1988).

${ }^{25}$ B. Lin, J. B. Peng, J. B. Ketterson, P. Dutta, B. N. Thomas, J. Buontempo, and S. A. Rice, J. Chem. Phys. 90, 2393 (1989).

${ }^{26}$ M. C. Shih, T. M. Bohanon, J. M. Mikrut, P. Zschack, and P. Dutta, J. Chem. Phys. 97, 4485 (1992).

${ }^{27}$ G. A. Overbeck, D. Honig, and D. Möbius, Langmuir 9, 555 (1993).

${ }^{28}$ W. J. Foster, M. C. Shih, and P. S. Pershan, J. Chem. Phys. 105, 3307 (1996).

${ }^{29}$ P. G. de Gennes, Rev. Mod. Phys. 57, 827 (1985).

${ }^{30} \mathrm{M}$. Schick, in Liquids at Interfaces, edited by J. Charvolin, J. F. Joanny, and J. Zinn-Justin (Elsevier, Amsterdam, 1990), Vol. XLVIII, p. 419.

${ }^{31}$ E. M. Blokhuis and B. Widom, Current Opinion in Colloid Interface Sci. 1, 424 (1996).

${ }^{32}$ R. A. Cowley, in Equilibrium Structure and Properties of Surfaces and Interfaces, edited by A. Gonis and G. M. Stocks (Plenum, New York, 1992), p. 1.

${ }^{33}$ H. Dosch, Int. J. Mod. Phys. 6, 2773 (1992).

${ }^{34}$ I. K. Robinson and D. J. Tweet, Rep. Prog. Phys. 55, 599 (1992).

${ }^{35}$ J. G. Dash, H. Fu, and J. S. Wettlaufer, Rep. Prog. Phys. 58, 115 (1994).

${ }^{36}$ X. Z. Wu, B. M. Ocko, H. Tang, E. B. Sirota, S. K. Sinha, and M. Deutsch, Phys. Rev. Lett. 75, 1332 (1995).

${ }^{37}$ O. M. Magnussen, B. M. Ocko, M. J. Regan, K. Penanen, P. S. Pershan, and M. Deutsch, Phys. Rev. Lett. 74, 4444 (1995).

${ }^{38}$ M. J. Regan, E. H. Kawamoto, S. Lee, P. S. Pershan, N. Maskil, M. Deutsch, O. M. Magnussen, B. M. Ocko, and L. E. Berman, Phys. Rev. Lett. 75, 2498 (1995).

${ }^{39}$ N. Lei, Z. Huang, and S. A. Rice, J. Chem. Phys. 104, 4802 (1996).

${ }^{40}$ D. Raoux, in Neutron and Synchrotron Radiation for Condensed Matter Studies, edited by J. Baruchel, J.-L. Hodeau, M. S. Lehmann, J.-R. Regnard, and C. Schlenker (Springer, New York, 1993), Vol. 1, pp. 37-78.

${ }^{41}$ H. Winick, Synchrotron Radiation Sources: A Primer (World Scientific, Singapore, 1994).

${ }^{42}$ B. I. Halperin, in Symmetries and Broken Symmetries in Condensed Mat- 
ter Physics: Proceedings of the Colloque Pierre Curie, edited by N. Boccara (IDSET, Paris, 1981).

${ }^{43}$ B. Berge, O. Konovalov, J. Lajzerowicz, A. Renault, J. P. Rieu, M. Vallade, J. Als-Nielsen, G. Grubel, and J. F. Legrand, Phys. Rev. Lett. 73, 1652 (1994).

${ }^{44}$ K. Kjaer, J. Als-Nielsen, C. A. Helm, L. A. Laschuber, and H. Möhwald, Phys. Rev. Lett. 58, 2224 (1987).

${ }^{45} \mathrm{~J}$. Als-Nielsen and H. Möwald, in Handbook of Synchrotron Radiation, edited by S. Ebashi, E. Rubenstein, and M. Koch (North-Holland, Amsterdam, 1991), Vol. 4, p. 1

${ }^{46}$ E. B. Sirota, J. Collett, P. S. Pershan, and L. B. Sorensen, Phys. Rev. Lett. 55, 2039 (1985).

${ }^{47}$ S. Amador, P. S. Pershan, H. Stragier, B. D. Swanson, D. J. Tweet, L. B. Sorensen, E. B. Sirota, G. E. Ice, and A. Habenschuss, Phys. Rev. A 39, 2703 (1989).

${ }^{48}$ C.-F. Chou, J. T. Ho, S. W. Hui, and V. Surendranth, Phys. Rev. Lett. 76, 4556 (1996)

${ }^{49}$ N. Lei, Z. Huang, and S. A. Rice, J. Chem. Phys. 105, 9615 (1996).

${ }^{50}$ J. D. Brock, A. Aharony, R. J. Birgeneau, K. W. Evans-Lutterodt, J. D. Litster, P. M. Horn, G. B. Stephenson, and A. R. Tajbakhsh, Phys. Rev. Lett. 57, 98 (1986).

${ }^{51}$ J. D. Shindler, E. A. L. Mol, A. Shalaginov, and W. H. d. Jeu, Phys. Rev. Lett. 74, 722 (1995).

${ }^{52}$ D. Vaknin, J. Y. Wang, and R. A. Uphaus, Langmuir 11, 1435 (1995).

${ }^{53} \mathrm{H}$. Eickenbusch and P. Härtwich, Fullerene: Analyse \& Bewertung Zukünftiger Technologien (VDI Technologiezentrum, Düsseldorf, 1993).

${ }^{54}$ R. Malhorta, D. F. McMillen, D. S. Tse, D. L. Lorents, R. S. Ruoff, and D.

M. Keegan, Energy and Fuels 7, 685 (1993).

${ }^{55}$ R. Baum, Chem. Eng. News 22, 8 (1993).

${ }^{56}$ Y. S. Obeng and A. J. Bard, J. Am. Chem. Soc. 113, 6279 (1991)

${ }^{57}$ N. C. Maliszewskyj, P. A. Heiney, D. R. Jones, R. M. Strongin, M. A. Cichy, I. Smith, and B. Amos, Langmuir 9, 1439 (1993).

${ }^{58}$ R. Back and R. B. Lennox, J. Phys. Chem. 96, 8149 (1992).

${ }^{59}$ J. Guo, Y. Xu, Y. Li, Y. Yao, D. Zhu, and C. Bai, Chem. Phys. Lett. 195, 625 (1992).

${ }^{60}$ J. Milliken, D. D. Dominguez, H. H. Nelson, and W. R. Barger, Chem. Mater. 4, 252 (1992).

${ }^{61}$ Y. Tomioka, M. Ishibashi, H. Kajiyama, and Y. Taniguchi, Langmuir 9, 32 (1993).
${ }^{62}$ J. Y. Wang, D. Vaknin, R. A. Uphaus, K. Kjaer, and M. Lösche, Thin Solid Films 242, 40 (1994).

${ }^{63}$ Note that the requirement that $\alpha=\beta$ and $\theta=0$ (see Fig. 1), which is the the condition for specular reflectivity, is synonymous with $Q_{x y}=0$.

${ }^{64}$ P. S. Pershan and J. Als-Nielsen, Phys. Rev. Lett. 52, 759 (1984).

${ }^{65}$ P. M. Chaikin and T. C. Lubensky, Principles of Condensed Matter Physics (Cambridge University Press, New York, 1995).

${ }^{66}$ Strictly speaking, statistical fluctuations destroy the long-range positional order in 2D crystals; however, unless special procedures are invoked it is difficult to distinguish between the singular "power law" line shape of a $2 \mathrm{D}$ crystal and the superposition of a resolution limited Bragg peak on a thermal background, as is observed in 3D. See Berge et al., Phys. Rev. Lett. 73, 1652 (1994).

${ }^{67}$ T. M. Fischer, R. F. Bruinsma, and C. M. Knobler, Phys. Rev. E 50, 413 (1994).

${ }^{68}$ S. Garoff, H. W. Deckman, J. H. Dunsmuir, and M. S. Alvarez, J. Phys. (Paris) 47, 701 (1986).

${ }^{69}$ J. Rudnick and R. Bruinsma, Phys. Rev. Lett. 74, 2491 (1995).

${ }^{70}$ J. Ruiz-Garcia, X. Qiu, M.-W. Tsao, G. Marshall, C. M. Knobler, G. A. Overbeck, and D. Mobius, J. Phys. Chem. 97, 6955 (1993).

${ }^{71}$ D. Jacquemain, S. G. Wolf, F. Leveiller, M. Deutsch, K. Kjaer, J. AlsNielsen, M. Lahav, and L. Leiserowitz, Angew. Chem. Int. Ed. Engl. 31, 130 (1992).

${ }^{72}$ C. M. Knobler, J. Phys. Condens. Matter 3, 1 (1991).

${ }^{73}$ S. Henon and J. Meunier, Rev. Sci. Instrum. 62, 936 (1991).

${ }^{74}$ P. S. Pershan, Phys. Rev. E 50, 2369 (1994).

${ }^{75}$ M. S. Dresselhaus, G. Dresselhaus, and P. C. Eklund, Science of Fullerenes and Carbon Nanotubes (Academic, San Diego, 1996).

${ }^{76}$ W. Kratschmer, L. D. Lamb, K. Fostiropoulos, and D. R. Huffman, Nature 347, 354 (1990).

${ }^{77}$ G. S. Cargill III, in Solid State Physics, edited by H. Ehrenreich, F. Seitz, and D. Turnbull (Academic, New York, 1975), Vol. 30, p. 227.

${ }^{78}$ P. A. Egelstaff, An Introduction to the Liquid State (Academic, New York, 1967).

${ }^{79}$ B. E. Warren, X-ray Diffraction (Addison-Wesley, Reading, 1969).

${ }^{80}$ D. K. Schwartz, M. L. Schlossman, E. H. Kawamoto, G. J. Kellogg, P. S. Pershan, and B. M. Ocko, Phys. Rev. A 41, 5687 (1990). 